\title{
Self-Adjoint Algebras of Unbounded Operators *
}

\author{
ROBERT T. POWERS $\star \star$ \\ University of Pennsylvania, Philadelphia, Pennsylvania
}

Received November 11, 1970

\begin{abstract}
Unbounded *-representations of *-algebras are studied. Representations called self-adjoint representations are defined in analogy to the definition of a self-adjoint operator. It is shown that for self-adjoint representations certain pathologies associated with commutant and reducing subspaces are avoided. A class of well behaved self-adjoint representations, called standard representations, are defined for commutative *-algebras. It is shown that a strongly cyclic self-adjoint representation of a commutative *-algebra is standard if and only if the representation is strongly positive, i.e., the representations preserves a certain order relation. Similar results are obtained for *-representations of the canonical commutation relations for a finite number of degrees of freedom.
\end{abstract}

\section{Introduction}

In this paper we study unbounded *-representations of *-algebras. The basic definitions, notation and motivation are drawn from the Wightman formulation of quantum field theory and the theory of Lie algebras. The general plan of the paper is to examine some of the pathologies associated with *-algebras of unbounded operators and, then, to find natural definitions which rule out these pathologies. Two such definitions are those of self-adjointness for representations (Section IV) and strong positivity (Sections VII and VIII).

The results of each section are summarized at the beginning of each section. We claim little or no originality for the contents of Sections I, II, V and VI which consist largely of background material, definitions modified from $C^{*}$-algebra theory and quantum field theory and known examples illustrating features of unbounded representations.

The author would like to thank Professors J. Kazden and S. Sakai for acquainting him with Hilbert's work on polynomials which are the sums of squares of real polynomials and Sherman's work on positive functionals on *-algebras of unbounded operators. The author would like to thank Professor O. Nielson for helpful discussions during the research for this paper.

* Work supported in part by U.S. Atomic Energy Commission under Contract AT(30-1)-2171 and by the National Science Foundation.

$\star \star$ Alfred P. Sloan Foundation Fellow.

7 Commun. math Phys, Vol. 21 


\section{Review of Known Results on Unbounded Operators}

We review some of the well known results on unbounded operators and refer to [3, Chap. XII], [14] and [16] as references for the results stated in this section. In this paper we will work exclusively with Hilbert spaces over the complex numbers. We use the physicists' inner product $(f, g)$ which is linear in $g$ and conjugate linear in $f$.

A bounded or unbounded operator $A$ on a Hilbert space $\mathfrak{G}$, defined on a linear manifold $\mathfrak{D}(A)$ (called the domain of $A$ ) is a linear mapping of $\mathfrak{D}(A)$ into $\mathfrak{H}$. Unless otherwise stated we assume $\mathfrak{D}(A)$ is dense in $\mathfrak{H}$. If $A$ and $B$ are operators on $\mathfrak{H}$ with domains $\mathfrak{D}(A)$ and $\mathfrak{D}(B)$ we say $A$ is an extension of $B$, denoted $A \supset B$, if $\mathfrak{D}(A) \supset \mathfrak{D}(B)$ and $A f=B f$ for all $f \in \mathfrak{D}(B)$. We denote by $G_{A}$ the graph of the operator $A$, the linear manifold in $\mathfrak{H} \oplus \mathfrak{H}$ of all pairs, $G_{A}=\{\{f, A f\} ; f \in \mathfrak{D}(A)\}$. Clearly $A \supset B$ if and only if $G_{A} \supset G_{B}$. An operator $A$ is said to be closed if its graph $G_{A}$ is closed, i.e. $A$ is closed if and only if $f_{n} \rightarrow f$ and $A f_{n} \rightarrow g$ as $n \rightarrow \infty$ implies $f \in \mathfrak{D}(A)$ and $A f=g$. We say $A$ is closable if $A$ has a closed extension. If $A$ is closable we denote by $\bar{A}$ the smallest closed extension of $A$, i.e. if $A$ is closable then $G_{\bar{A}}=\bar{G}_{A}$, where $\{\bar{S}\}$ denotes the closure of a set $S$ in a Hilbert space.

If $A$ is an operator with dense domain $\mathfrak{D}(A) \subset \mathfrak{H}$ we denote by $A^{*}$ the hermitian adjoint of $A$. The graph of $A^{*}$ is given by

$$
G_{A}=\{\{f, g\} \in \mathfrak{H} \oplus \mathfrak{H} ;(f, A h)=(g, h) \text { for all } h \in \mathfrak{D}(A)\} .
$$

The domain of $A^{*}$ consists of all those vectors satisfying the relation,

$$
\begin{aligned}
\mathfrak{D}\left(A^{*}\right)=\{f \in \mathfrak{H} ;|(f, A h)| \leqq K\|h\| & \text { for some constant } K \geqq 0 \\
& \text { and all } h \in \mathfrak{D}(A)\} .
\end{aligned}
$$

$A^{*}$ is always a closed operator. However, the domain $\mathfrak{D}\left(A^{*}\right)$ may not be dense in $\mathfrak{H}$. In fact $A$ is closable if and only if $\mathfrak{D}\left(A^{*}\right)$ is dense in $\mathfrak{H}$ and if $\mathfrak{D}\left(A^{*}\right)$ is dense in $\mathfrak{H}$ then $\bar{A}=A^{* *}$.

An operator $A$ is said to be hermitian if $A^{*} \supset A$. An operator $A$ is hermitian if and only if $(f, A g)=(A f, g)$ for all $f, g \in \mathfrak{D}(A)$. Every hermitian operator is closable since $\mathfrak{D}\left(A^{*}\right) \supset \mathfrak{D}(A)$ and hence $\mathfrak{D}\left(A^{*}\right)$ is dense in $\mathfrak{H}$. An operator $A$ is self-adjoint if $A=A^{*}$. An hermitian operator $A$ is self-adjoint if and only if $\mathfrak{D}(A)=\mathfrak{D}\left(A^{*}\right)$. Since $A^{*}$ is closed it follows that every self-adjoint operator is closed. An operator $A$ is said to be essentially self-adjoint if its closure $\bar{A}$ is self-adjoint.

If $A$ is an hermitian operator we denote by $\mathfrak{D}_{+}$and $\mathfrak{D}_{-}$the deficiency spaces of $A$ defined by the relations,

$$
\begin{aligned}
& \mathfrak{D}_{+}=\{\operatorname{Range}(A+i I)\}^{\perp}=\left\{f \in \mathfrak{D}\left(A^{*}\right) ; A^{*} f=i f\right\}, \\
& \mathfrak{D}_{-}=\{\operatorname{Range}(A-i I)\}^{\perp}=\left\{f \in \mathfrak{D}\left(A^{*}\right) ; A^{*} f=-i f\right\},
\end{aligned}
$$


where $\{S\}^{\perp}$ denotes the orthogonal complement of $S$. A useful result of von Neumann states that an hermitian operator $A$ is essentially selfadjoint if and only if $\mathfrak{D}_{+}=\mathfrak{D}_{-}=\{0\}$. Furthermore, $A$ has a self-adjoint extension if and only if the dimension of $\mathfrak{D}_{+}$equals the dimension of $\mathfrak{D}_{-}$.

If $A$ is a self-adjoint operator then $A$ has a unique spectral resolution $\{E(\lambda) ;-\infty<\lambda<+\infty\}$ such that $E(\lambda) E(\mu)^{*}=E(\min \{\lambda, \mu\}), E(\lambda) \rightarrow E(\mu)$ (strongly) as $\lambda \rightarrow \mu$ from above, $E(\lambda) \rightarrow I$ (strongly) as $\lambda \rightarrow+\infty, E(\lambda) \rightarrow 0$ (strongly) as $\lambda \rightarrow-\infty$.

and

$$
(f, A g)=\int \lambda d(f, E(\lambda) g) \text { for all } f \in \mathfrak{H} \text { and } g \in \mathfrak{D}(A)
$$

$$
\mathfrak{D}(A)=\left\{f \in \mathfrak{H} ; \int\left(\lambda^{2}+1\right) d(f, E(\lambda) f)<\infty\right\} .
$$

If $A$ is an hermitian operator and $C$ is a bounded operator we say $C$ commutes with $A$ if

$$
(f, C A g)=(A f, C g) \text { for all } f, g \in \mathfrak{D}(A) .
$$

If $A$ is essentially self-adjoint then $C$ commutes with $A$ if and only if $C$ commutes with the spectral projections of $\bar{A}$ (see [6, Chap. VIII, Sec. 120] or [16, Chap. IV, and 17, Sec. 4, Thm. VII, p. 251]).

Finally, we state some results concerning normal operators (see, e.g. [3, Chap. XII, Problems 9, 10, 11 and 12, p. 1258-1259]). If $A$ is a closed operator then $A^{*} A$ is self-adjoint where $\mathfrak{D}\left(A^{*} A\right)=\{f \in \mathfrak{D}(A)$; $\left.A f \in \mathfrak{D}\left(A^{*}\right)\right\}, A A^{*}$ is also self-adjoint. A closed operator $A$ is said to be normal if $A^{*} A=A A^{*}$. A closed operator $A$ is normal if and only if $\mathfrak{D}(A)=\mathfrak{D}\left(A^{*}\right)$ and $\|A f\|=\left\|A^{*} f\right\|$ for all $f \in \mathfrak{D}(A)$. If $A$ is normal then $A_{1}=(1 / 2)\left(A+A^{*}\right)$ and $A_{2}=(1 / 2 i)\left(A-A^{*}\right)$ are essentially self-adjoint on $\mathfrak{D}(A)$ and the spectral resolutions of $\bar{A}_{1}$ and $\bar{A}_{2}$ commute.

\section{Closed *-Representations of *-Algebras}

In this section we define *-representations of *-algebras. For need of an adjective we will call *-representations hermitian representations. In analogy with the notion of a closed operator we define the notion of a closed representation. We show that just as every hermitian operator has a minimal closed extension, every hermitian representation of a *-algebra has a minimal closed extension.

A *-algebra $\mathfrak{U}$ is an algebra over the complex numbers with a *-operator satisfying;

(i) $A^{* *}=A$,

(ii) $(\alpha A+B)^{*}=\bar{\alpha} A^{*}+B^{*}$,

(iii) $(A B)^{*}=B^{*} A^{*}$ 
for all $A, B \in \mathfrak{A}$ and complex numbers $\alpha$. In general $\mathfrak{A}$ will not be normed since we are interested in the study of unbounded *-representations.

All algebras in this paper will have a unit denoted by I .

Definition 2.1. A representation $\pi$ of an algebra $\mathfrak{U}$ on a Hilbert space $\mathfrak{H}$ is a mapping of $\mathfrak{U}$ into linear operators all defined on a common dense domain $\mathfrak{D}(\pi)$ which is dense in $\mathfrak{H}$ and $\pi$ satisfies the conditions, $\pi(I)=I$, the unit operator on $\mathfrak{H}$,

(i) $\pi(\alpha A+B) f=\alpha \pi(A) f+\pi(B) f$, for all $A, B \in \mathfrak{A} f \in \mathfrak{D}(\pi)$ and all complex numbers $\alpha$.

(ii) $\pi(A) \mathfrak{D}(\pi) \subset \mathfrak{D}(\pi)$ for all $A \in \mathfrak{A}$ and $\pi(A) \pi(B) f=\pi(A B) f$ for all $A, B \in \mathfrak{U}$ and $f \in \mathfrak{D}(\pi)$.

Definition 2.2. A representation $\pi$ of a $*$-algebra $\mathfrak{A}$ on a Hilbert space $\mathfrak{H}$ is said to be hermitian or a $*$-representation if

(iii) $(f, \pi(A) g)=\left(\pi\left(A^{*}\right) f, g\right)$ for all $f, g \in \mathfrak{D}(\pi)$ and $A \in \mathfrak{A}$, i.e. $\pi(A)^{*}$ $\supset \pi\left(A^{*}\right)$ for all $A \in \mathfrak{U}$.

We remark that a representation $\pi$ is hermitian if and only if for every hermitian $A \in \mathfrak{A}$ (i.e. $\left.A=A^{*}\right) \pi(A)$ is hermitian.

This definition of a ${ }^{*}$-representation is well known to workers in the theory of representations of Lie algebras and axiomatic quantum field theory (see e.g. [15]). In the Wightman formulation of quantum field theory one assumes that the "smeared fields $\Phi(f)$ " generate a *-algebra and a field theory is a cyclic *-representation of this algebra satisfying additional assumptions such as Lorentz invariance and local commutativity.

Definition 2.3. If $\pi_{1}$ and $\pi_{2}$ are representations of an algebra $\mathfrak{A}$ on a Hilbert space $\mathfrak{H}$ we say $\pi_{1}$ is an extension of $\pi_{2}$, denoted $\pi_{1} \supset \pi_{2}$, if $\mathfrak{D}\left(\pi_{1}\right) \supset \mathfrak{D}\left(\pi_{2}\right)$ and $\pi_{1}(A) \supset \pi_{2}(A)$ for all $A \in \mathfrak{A}$.

If $\pi$ is a representation of an algebra $\mathfrak{A}$ on a Hilbert space $\mathfrak{H}$ with domain $\mathfrak{D}(\pi)$, there is a natural induced topology on $\mathfrak{D}(\pi)$. This topology is defined as follows. Suppose $S$ is a finite set of elements of $\mathfrak{A}$. We define the semi-norm $\|\cdot\|_{S}$ on $\mathfrak{D}(\pi)$ as

$$
\|f\|_{S}=\sum_{A \in S}\|\pi(A) f\|
$$

where $\|f\|$ is the Hilbert space norm of $f$. Note $S \supset S^{\prime}$ implies $\|f\|_{S} \geqq\|f\|_{S}$, for all $f \in \mathfrak{D}(\pi)$. We define the induced topology on $\mathfrak{D}(\pi)$ as the topology generated by the neighborhoods,

$$
\mathfrak{N}(f ; S, \varepsilon)=\left\{g \in \mathfrak{D}(\pi) ;\|f-g\|_{S}<\varepsilon\right\} .
$$

Note $\pi(A)$ is a continuous mapping of $\mathfrak{D}(\pi)$ into $\mathfrak{D}(\pi)$ in the induced topology for all $A \in \mathfrak{U}$. 
Just as there is a notion of a closed operator there is an analogous notion of a closed representation.

Definition 2.4. We say $\pi$ is a closed representation of $\mathfrak{A}$ if $\mathfrak{D}(\pi)$ is complete in the induced topology.

We show that every hermitian representation of a *algebra can be extended to a closed hermitian representation. The following lemma will be useful.

Lemma 2.5. Suppose $\pi$ is $a^{*}$-representation of $a{ }^{*}$-algebra and $S \subset \mathfrak{A}$ is a finite set of elements of $\mathfrak{A}$. Then, there is an hermitian element $C \in \mathfrak{A}$ such that $\|\pi(C) f\| \geqq\|f\|_{S}$ for all $f \in \mathfrak{D}(\pi)$.

Proof. Let $S=\left\{A_{1}, A_{2}, \ldots, A_{n}\right\}$ be a finite subset of $\mathfrak{A}$. Let

$$
D=(n / 2) \sum_{i=1}^{n} A_{i}^{*} A_{i} \quad \text { and } \quad C=D+I .
$$

We have

$$
\begin{aligned}
\|\pi(C) f\|^{2} & =\|\pi(D) f\|^{2}+2(f, \pi(D) f)+\|f\|^{2} \geqq n \sum_{i=1}^{n}\left(f, \pi\left(A_{i}^{*} A_{i}\right) f\right) \\
& =n \sum_{i=1}^{n}\left\|\pi\left(A_{i}\right) f\right\|^{2} \geqq\left(\sum_{i=1}^{n}\left\|\pi\left(A_{i}\right) f\right\|\right)^{2} \geqq\|f\|_{S}^{2} \text {, for all } f \in \mathfrak{D}(\pi) .
\end{aligned}
$$

This completes the proof of the lemma.

Lemma 2.6. Suppose $\pi$ is a*-representation of $a *$-algebra $\mathfrak{A}$ on a Hilbert space $\mathfrak{h}$. Then, there is a unique minimal closed extension $\pi$ of $\pi$. Furthermore, the domain of $\pi$ is given by

$$
\mathfrak{D}(\pi)=\bigcap_{A \in \mathfrak{A}} \mathfrak{D}(\overline{\pi(A)})
$$

and $\pi(A) f=\overline{\pi(A)} f$ for all $A \in \mathfrak{A}$ and all $f \in \mathfrak{D}(\pi)$.

Proof. Suppose $\pi$ is a *-representation of a *-algebra $\mathfrak{A}$ on a Hilbert space $\mathfrak{H}$. Since $\pi$ is hermitian we have $\pi(A)^{*} \supset \pi\left(A^{*}\right)$ for each $A \in \mathfrak{H}$. Hence, $\pi(A)$ is closable for each $A \in \mathfrak{A}$. Let $\mathfrak{D}(\pi)$ be defined

$$
\mathfrak{D}(\pi)=\bigcap_{A \in \mathfrak{Q}} \mathfrak{D} \overline{(\pi(A))}
$$

and let $\pi(A)=\overline{\pi(A)} \mid \mathfrak{D}(\pi)$ for all $A \in \mathfrak{A}$. We show $\pi$ is a closed *-representation of $\mathfrak{U}$. Clearly, $\pi$ is linear. We show $\pi(A) \mathfrak{D}(\pi) \subset \mathfrak{D}(\pi)$ for all $A \in \mathfrak{A}$ and $\pi(A B) f=\pi(A) \pi(B) f$ for all $f \in \mathfrak{A}(\pi)$ and $A, B \in \mathfrak{A}$.

Suppose $A, B \in \mathfrak{A}$. From Lemma 2.5 it follows that there is a $C \in \mathfrak{A}$ such that

$$
\|\pi(C) f\| \geqq\|\pi(A B) f\|+\|\pi(B) f\|+\|f\|
$$


for all $f \in \mathfrak{D}(\pi)$. Suppose $f \in \mathfrak{D}(\pi)$. We have $f \in \mathfrak{D}(\overline{\pi(C)})$. Hence, there is a sequence $\left\{f_{n} \in \mathfrak{D}(\pi) ; n=1,2, \ldots\right\}$ such that $\pi(C) f_{n} \rightarrow \overline{\pi(C)} f$ as $n \rightarrow \infty$. It follows from the above inequality that

(a) $\left\|f_{n}-f\right\| \rightarrow 0$,

(b) $\left\|\pi(B)\left(f_{n}-f_{m}\right)\right\| \rightarrow 0$,

(c) $\left\|\pi(A) \pi(B)\left(f_{n}-f_{m}\right)\right\|=\left\|\pi(A B)\left(f_{n}-f_{m}\right)\right\| \rightarrow 0$

as $n, m \rightarrow \infty$. From (a) and (b) it follows that $f \in \mathfrak{D}(\overline{\pi(B)})$ and

(d) $\left\|\overline{\pi(B)}\left(f_{n}-f\right)\right\| \rightarrow 0$

as $n \rightarrow \infty$. From (c) and (d) it follows that $\overline{\pi(B)} f \in \mathfrak{D}(\overline{\pi(A))}$. From (a) and (c) it follows that $f \in \mathfrak{D}(\overline{\pi(A B)})$ and $\overline{\pi(A B)} f=\overline{\pi(A)} \overline{\pi(B)} f$. Since $A \in \mathfrak{A}$ is arbitrary it follows that

$$
\pi(B) f=\overline{\pi(B)} f \in \bigcap_{A \in \mathfrak{I}} \mathfrak{D}(\overline{\pi(A)})=\mathfrak{D}(\pi) .
$$

Hence, $\pi(B) \mathfrak{D}(\pi) \subset \mathfrak{D}(\pi)$ for all $B \in \mathfrak{A}$ and $\pi(A B) f=\pi(A) \pi(B) f$ for all $A, B \in \mathfrak{A}$ and all $f \in \mathfrak{D}(\pi)$.

We show $\pi$ is hermitian. Suppose $A \in \mathfrak{A}$ and $f, g \in \mathfrak{D}(\pi)$. Since $f \in \mathfrak{D}\left(\overline{\pi\left(A^{*}\right)}\right)$ and $g \in \mathfrak{D}\left(\overline{\pi(A))}\right.$ there are sequences $\left\{f_{n}, g_{n} \in \mathfrak{D}(\pi) ; n=1,2, \ldots\right\}$ such that $f_{n} \rightarrow f, g_{n} \rightarrow g, \pi\left(A^{*}\right) f_{n} \rightarrow \overline{\pi\left(A^{*}\right)} f$ and $\pi(A) g_{n} \rightarrow \overline{\pi(A)}$ as $n \rightarrow \infty$. Using these sequences we obtain the relation.

$$
\begin{aligned}
(f, \pi(A) g) & =\lim _{n \rightarrow \infty}\left(f_{n}, \pi(A) g_{n}\right) \\
& =\lim _{n \rightarrow \infty}\left(\pi\left(A^{*}\right) f_{n}, g_{n}\right)=\left(\pi\left(A^{*}\right) f, g\right)
\end{aligned}
$$

for all $A \in \mathfrak{A}$ and all $f, g \in \mathfrak{D}(\pi)$. Hence, $\pi$ is hermitian.

We show $\pi$ is closed. Suppose $\left\{f_{\alpha} ; \alpha \in I_{0}\right\}$ is a Cauchy net in $\mathfrak{D}(\pi)$ in the induced topology on $\mathfrak{D}(\pi)$. Then $\left\{\pi(A) f_{\alpha} ; \alpha \in I_{0}\right\}$ is a Cauchy net in $\mathfrak{H}$ for each $A \in \mathfrak{A}$. Since $\pi(A)$ is a closable operator for each $A \in \mathfrak{Q}$ there is an $f \in \mathfrak{D} \overline{(\pi(A))}=\mathfrak{D}(\overline{\pi(A)})$ such that $\lim _{\alpha} f_{\alpha}=f$ and $\lim _{\alpha} \pi(A) f_{\alpha}=\overline{\pi(A)} f$. Hence, $f \in \mathfrak{D}(\pi)$ and $\lim _{\alpha}\left\|\pi(A)\left(f_{\alpha}-f\right)\right\|^{\alpha}=0$ for all $A \in \mathfrak{\alpha} \mathfrak{U}$. Hence, $\mathfrak{D}(\pi)$ is complete in the induced topology and $\pi$ is closed.

Finally, we show $\pi$ is the minimal closed extension of $\pi$. Suppose $\pi_{1}$ is a closed extension of $\pi$. We show $\pi_{1} \supset \pi$. Suppose $f_{0} \in \mathfrak{D}(\pi)$. We show $f_{0} \in \mathfrak{D}\left(\pi_{1}\right)$ and $\pi_{1}(A) f_{0}=\pi(A) f_{0}$ for all $A \in \mathfrak{U}$.

Suppose $S$ is a finite subset of $\mathfrak{A}$ and $\varepsilon>0$. From Lemma 2.5 it follows that there is a $C \in \mathfrak{A}$ such that

$$
\|\pi(C) f\| \geqq \sum_{A \in S}\|\pi(A) f\| \text { for all } f \in \mathfrak{D}(\pi) .
$$

Since $f_{0} \in \mathfrak{D}(\pi) \subset \mathfrak{D}(\overline{\pi(C)})$ there is a vector $f \in \mathfrak{D}(\pi)$ such that $\| \overline{\pi(C)} f_{0}$ $-\pi(C) f \|<\varepsilon$. Hence, for each finite subset $S$ of $\mathfrak{A}$ and $\varepsilon>0$ the set

$$
Q(S, \varepsilon)=\left\{f \in \mathfrak{D}(\pi) ; \sum_{A \in S}\left\|\pi(A) f-\pi(A) f_{0}\right\|<\varepsilon\right\}
$$


is not empty. Let $I_{0}$ be the set of all pairs $\{S, \varepsilon\}$ partially ordered by the relation $\alpha=\{S, \varepsilon\}<\left\{S^{\prime}, \varepsilon^{\prime}\right\}=\alpha^{\prime}$ if and only if $S^{\prime} \supset S$ and $\varepsilon^{\prime} \leqq \varepsilon$. By the axiom of choice there is a net $\left\{f_{\alpha} \in Q(\alpha) ; \alpha \in I_{0}\right\}$. Clearly, this net is a Cauchy net in the induced topology on $\mathfrak{D}(\pi)$ and since $\pi_{1} \supset \pi$ this net is a Cauchy net in the induced topology on $\mathfrak{D}\left(\pi_{1}\right)$. Since $\lim _{\alpha} \pi_{1}(A) f_{\alpha}$ $=\overline{\pi(A)} f_{0}=\pi(A) f_{0}$ for all $A \in \mathfrak{A}$ and $\pi_{1}$ is closed it follows that $f_{0} \in \mathfrak{D}\left(\pi_{1}\right)$ and $\pi_{1}(A) f_{0}=\pi(A) f_{0}$ for all $A \in \mathfrak{U}$. Hence, we have $\pi_{1} \supset \pi$. This completes the proof of the theorem.

Hereafter, we denote by $\pi$ the minimal closed extension of the *-representation $\pi$.

We remark that if $\mathfrak{A}$ is a Banach *-algebra (i.e., a complete normed *-algebra) then a *-representation $\pi$ of $\mathfrak{A}$ on a Hilbert space $\mathfrak{H}$ is closed if and only if $\mathfrak{D}(\pi)=\mathfrak{H}$. We also note that if $\pi$ is a ${ }^{*}$-representation of a *-algebra $\mathfrak{U}$ and $\mathfrak{D}(\pi)=\mathfrak{H}$ then $\pi(\mathfrak{U})$ is a *-algebra of bounded operators. This follows from the closed graph theorem.

\section{The Commutant $\pi(\mathfrak{l})^{\prime}$}

We define the commutant $\pi(\mathfrak{A})^{\prime}$ of a *-algebra $\pi(\mathfrak{A})$ of unbounded operators. Our definition corresponds to the definition used in the Wightman formulation of quantum field theory (see [15, Eq. (3-8), p. 101]). The commutant $\pi(\mathfrak{2})^{\prime}$ is a weakly closed symmetric linear subset of $\mathfrak{B}(\mathfrak{H})$ (all bounded operators on $\mathfrak{G}$ ). The commutant need not be an algebra as shown in Lemma 3.2.

Definition 3.1. Suppose $\pi$ is a *-representation of a *-algebra $\mathfrak{A}$ on a Hilbert space $\mathfrak{H}$. The commutant of $\pi(\mathfrak{Q})$, denoted $\pi(\mathfrak{A})^{\prime}$, consists of all bounded operators $C$ on $\mathfrak{S}$ such that

$$
(f, C \pi(A) g)=\left(\pi\left(A^{*}\right) f, C g\right)
$$

for all $f, g \in \mathfrak{D}(\pi)$ and $A \in \mathfrak{A}$.

If $\pi(\mathfrak{A})$ is a *-algebra of bounded operators $\pi(\mathfrak{A})^{\prime}$ is simply the commutant of $\pi(\mathfrak{Q})$ defined in the usual manner. The above definition of the commutant is essentially the weakest definition, i.e., this definition gives the biggest commutant.

One can easily check that the commutant $\pi(\mathfrak{H})^{\prime}$ of a *-representation $\pi$ has the following properties.

(i) $\pi(\mathfrak{A})^{\prime}$ is a complex linear manifold,

(ii) $\pi(\mathfrak{H})^{\prime}$ is symmetric, i.e., $C \in \pi(\mathfrak{H})^{\prime} \Rightarrow C^{*} \in \pi(\mathfrak{H})^{\prime}$,

(iii) $\pi(\mathfrak{U})^{\prime}$ is closed in the weak operator topology,

(iv) If $\pi$ is the closure of $\pi$ then $\pi(\mathfrak{H})^{\prime}=\pi(\mathfrak{H})^{\prime}$.

In general $\pi(\mathfrak{H})^{\prime}$ is not an algebra as the next lemma shows. 
Lemma 3.2. Let $\mathfrak{A}$ be the free commutative algebra on one hermitian generator $A_{0}$ (i.e., $\mathfrak{U}$ consists of all polynomials $P=\alpha_{0} I+\alpha_{1} A_{0}+\alpha_{n} A_{0}^{n}$ with complex coefficients with multiplication and the ${ }^{*}$-operation defined in the obvious manner). Let $\pi$ be $a{ }^{*}$-representation of $\mathfrak{A}$ on a Hilbert space $\mathfrak{H}$. Then, $\pi(\mathfrak{U})^{\prime}$ is an algebra if and only if $\pi\left(A_{0}\right)$ is essentially self-adjoint.

Proof. Let $\mathfrak{A}$ and $\pi$ be as described in the lemma. We begin by showing that $\pi(\mathfrak{Q})^{\prime}$ consists of those bounded operators which commute with $\pi\left(A_{0}\right)$, i.e., those operators $C$ such that

(a) $\left(f, C \pi\left(A_{0}\right) g\right)=\left(\pi\left(A_{0}\right) f, C g\right)$ for all $f, g \in \mathfrak{D}(\pi)$.

Clearly, if $C \in \pi(\mathfrak{Q})^{\prime}$ then $C$ satisfies relation (a). Conversely, if $C$ satisfies relation (a) then

$$
\begin{aligned}
\left(f, C \pi\left(A_{0}^{n}\right) g\right) & =\left(f, C \pi\left(A_{0}\right) \pi\left(A_{0}^{n-1}\right) g\right)=\left(\pi\left(A_{0}\right) f, C \pi\left(A_{0}\right) \pi\left(A_{0}^{n-2}\right) g\right) \\
& =\cdots=\left(\pi\left(A_{0}^{n}\right) f, C g\right)
\end{aligned}
$$

for all $n=1,2, \ldots$ and all $f, g \in \mathfrak{D}(\pi)$. Since each element of $\mathfrak{A}$ is a polynomial in $A_{0}$ it follows that $C \in \pi(\mathfrak{R})^{\prime}$.

Now suppose that $\pi\left(A_{0}\right)$ is essentially self-adjoint. Let $\{E(\lambda)$; $<\infty<\lambda<+\infty\}$ be the spectral resolution of $\overline{\pi\left(A_{0}\right)}$. By the remarks in Section I a bounded operator $C$ commutes with $\pi\left(A_{0}\right)$ (i.e., $C$ satisfies relation (a)) if and only if $C E(\lambda)=E(\lambda) C$ for all $-\infty<\lambda<+\infty$. Hence, $\pi(\mathfrak{U})^{\prime}=\{E(\lambda) ;-\infty<\lambda<+\infty\}^{\prime}$ and $\pi(\mathfrak{2})^{\prime}$ is a von Neumann algebra.

Next suppose $\pi(\mathfrak{M})^{\prime}$ is an algebra. We begin by showing that the Cayley transform of $\overline{\pi\left(A_{0}\right)}$ is in $\pi(\mathfrak{A})^{\prime}$. Let $U$ be the partial isometry from Range $\overline{\left(\pi\left(A_{0}+i I\right)\right)}$ onto Range $\overline{\left(\pi\left(A_{0}-i I\right)\right)}$ defined by

$$
U f=\overline{\pi\left(A_{0}-i I\right)} g \quad \text { where } f=\overline{\pi\left(A_{0}+i I\right)} g .
$$

Note $U f=0$ for $f \in \operatorname{Range} \overline{\left(\pi\left(A_{0}+i I\right)\right)^{\perp}}$. Note $U^{*}$ is a partial isometry from Range $\overline{\left(\pi\left(A_{0}-i I\right)\right)}$ to Range $\overline{\left(\pi\left(A_{0}+i I\right)\right)}$ defined by

$$
U^{*} f=\overline{\pi\left(A_{0}+i I\right)} g \quad \text { where } f=\overline{\pi\left(A_{0}-i I\right)} g .
$$

We have that $U^{*} U=E_{+}$and $U U^{*}=E_{-}$where $E_{+}$and $E_{-}$are the hermitian projections onto Range $\overline{\left(\pi\left(A_{0}+i I\right)\right)}$ and Range $\overline{\left(\pi\left(A_{0}-i I\right)\right)}$, respectively.

We show $U \in \pi(\mathfrak{R})^{\prime}$. From the definition of $U$ it follows that

$$
\begin{aligned}
\left(f, U \pi\left(A_{0}\right) g\right) & =\left(f, U \pi\left(A_{0}+i I\right) g\right)-i(f, U g) \\
& =\left(f, \pi\left(A_{0}-i I\right) g\right)-i(f, U g)
\end{aligned}
$$


and

$$
\begin{aligned}
\left(\pi\left(A_{0}\right) f, U g\right) & =\left(U^{*} \pi\left(A_{0}\right) f, g\right) \\
& =\left(U^{*} \pi\left(A_{0}-i I\right) f, g\right)-i\left(U^{*} f, g\right) \\
& =\left(\pi\left(A_{0}+i I\right) f, g\right)-i(f, U g) \\
& =\left(f, \pi\left(A_{0}-i I\right) g\right)-i(f, U g), \text { for all } f, g \in \mathfrak{D}(\pi) .
\end{aligned}
$$

Hence, $U \in \pi(\mathfrak{A})^{\prime}$ and since $\pi(\mathfrak{A})^{\prime}$ is symmetric $U^{*} \in \pi(\mathfrak{A})^{\prime}$. Since $\pi(\mathfrak{A})^{\prime}$ is an algebra we have $I-E_{+}=I-U^{*} U$ and $I-E_{-}=I-U U^{*}$ are contained in $\pi(\mathfrak{Q})^{\prime}$. It follows that

$$
\begin{aligned}
\left(f,\left(I-E_{+}\right) g\right) & =\left(f,\left(I-E_{+}\right) g\right)+i\left(f,\left(I-E_{+}\right) \pi\left(A_{0}+i I\right) g\right) \\
& =i\left(f,\left(I-E_{+}\right) \pi\left(A_{0}\right) g\right) \\
& =i\left(\pi\left(A_{0}\right) f,\left(I-E_{+}\right) g\right) \\
& =i\left(\left(I-E_{+}\right) \pi\left(A_{0}+i I\right) f, g\right)-\left(f,\left(I-E_{+}\right) g\right) \\
& =-\left(f,\left(I-E_{+}\right) g\right)
\end{aligned}
$$

for all $f, g \in \mathfrak{D}(\pi)$. Since $\mathfrak{D}(\pi)$ is dense in $\mathfrak{H}$ we have $E_{+}=I$. A simimilar calculation shows $E_{-}=I$. Hence, $\pi\left(A_{0}\right)$ has deficiency spaces $\mathfrak{D}_{+}=\mathfrak{D}_{-}=\{0\}$. Hence, if $\pi(\mathfrak{R})^{\prime}$ is an algebra $\pi\left(A_{0}\right)$ is essentially self-adjoint. This completes the proof of the lemma.

\section{Self-Adjoint Representations of *-Algebras}

To each *-representation $\pi$ of a $*$-algebra $\mathfrak{A}$ there is a naturally associated adjoint representation $\pi^{*}$ of $\mathfrak{U}$. The domain and action of $\pi^{*}$ are given by

and

$$
\mathfrak{D}\left(\pi^{*}\right)=\bigcap_{A \in \mathfrak{Q}} \mathfrak{D}\left(\pi(A)^{*}\right)
$$

$$
\pi^{*}(A) f=\pi\left(A^{*}\right)^{*} f \text { for all } f \in \mathfrak{D}\left(\pi^{*}\right) .
$$

Just as the hermitian adjoint of a hermitian operator may fail to be hermitian, the hermitian adjoint representation $\pi^{*}$ may fail to be hermitian. In analogy with the definition of a self-adjoint operator we define self-adjoint representations as those hermitian representations which are equal to their adjoints (i.e. $\pi=\pi^{*}$ ). A *-representation $\pi$ is self-adjoint if and only if $\mathfrak{D}(\pi)=\mathfrak{D}\left(\pi^{*}\right)$. We show that just as every hermitian extension $A_{1}$ of an hermitian operator $A$ is an hermitian restriction of $A^{*}$, so is every hermitian extension $\pi_{1}$ of an hermitian representation $\pi$ an hermitian restriction of $\pi^{*}$. 
It is easily seen that for *-representations $\pi$ each operator $C \in \pi(\mathfrak{A})^{\prime}$ maps $\mathfrak{D}(\pi)$ into $\mathfrak{D}\left(\pi^{*}\right)$. It will follow that the commutant of a self-adjoint representation is necessarily a von Neumann algebra.

We next examine the problem of reducing subspaces for *-representations. If $\pi$ is a ${ }^{*}$-representation of a $C^{*}$-algebra $\mathfrak{A}$ on a Hilbert space $\mathfrak{H}$ and $\mathfrak{M} \subset \mathfrak{H}$ is a linear manifold which reduces $\pi$ (i.e. $\pi(A) \mathfrak{M} \subset \mathfrak{M}$ for all $A \in \mathfrak{U}$ ) then the hermitian projection $E_{\mathfrak{M}}$ onto the closure of $\mathfrak{M}$ is in the commutant, $\pi(\mathfrak{H})^{\prime}$. This is not true in general for unbounded *-representations as will be shown by a simple example. However, we will show that if $\pi$ is a self-adjoint representation of a $*$-algebra $\mathfrak{A}$ on a Hilbert space $\mathfrak{H}$ and $\mathfrak{M} \subset \mathfrak{D}(\pi)$ is a reducing subspace for $\pi$ such that $\pi$ restricted to $\mathfrak{M}$ is self-adjoint then the hermitian projection $E_{\mathfrak{M}}$ onto the closure of $\mathfrak{M}$ is in the commutant $\pi(\mathfrak{Q})^{\prime}$. Conversely, if $E \in \pi(\mathfrak{R})^{\prime}$ is an hermitian projection then $\mathfrak{M}=E \mathfrak{D}(\pi)$ is a reducing subspace for $\pi$ such that $\pi$ restricted to $\mathfrak{M}$ is self-adjoint. Hence, for self-adjoint representations there is a one-to-one correspondence between reducing self-adjoint subspaces and hermitian projections $E \in \pi(\mathfrak{H})^{\prime}$.

Finally we introduce the notation of when one algebra dominates another algebra and show that a *-representation $\pi$ is self-adjoint if $\pi(\mathfrak{l})$ contains a dominating subalgebra which is self-adjoint.

Lemma 4.1. Suppose $\pi$ is a *-representation of *-algebra $\mathfrak{A}$ on a Hilbert space $\mathfrak{H}$. Let

and let

$$
\mathfrak{D}\left(\pi^{*}\right)=\bigcap_{A \in \mathfrak{Q}} \mathfrak{D}\left(\pi(A)^{*}\right)
$$

$$
\pi^{*}(A)=\pi\left(A^{*}\right)^{*} \mid \mathfrak{D}\left(\pi^{*}\right) \text { for all } A \in \mathfrak{U} .
$$

Then, $\pi^{*}$ is a closed representation of $\mathfrak{A}\left(\pi^{*}\right.$ might not be a*-representation) which extends $\pi$. We call $\pi^{*}$ the hermitian adjoint of $\pi$.

Proof. Let $\pi^{*}$ be defined as in the lemma. We show that $\pi^{*}(B) \mathfrak{D}\left(\pi^{*}\right)$ $C \mathfrak{D}\left(\pi^{*}\right)$ and $\pi^{*}(A B) f=\pi^{*}(A) \pi^{*}(B) f$ for all $A, B \in \mathfrak{A}$ and $f \in \mathfrak{D}\left(\pi^{*}\right)$. Suppose $f \in \mathfrak{D}\left(\pi^{*}\right)$ and $g \in \mathfrak{D}(\pi)$. We have

$$
\begin{aligned}
\left(\pi\left(A^{*}\right) g, \pi^{*}(B) f\right) & =\left(\pi\left(A^{*}\right) g, \pi\left(B^{*}\right)^{*} f\right) \\
& =\left(\pi\left(B^{*}\right) \pi\left(A^{*}\right) g, f\right) \\
& =\left(\pi\left((A B)^{*}\right) g, f\right) \\
& =\left(g, \pi\left((A B)^{*}\right)^{*} f\right) \\
& =\left(g, \pi^{*}(A B) f\right) .
\end{aligned}
$$

Since $\left|\left(\pi\left(A^{*}\right) g, \pi^{*}(B) f\right)\right| \leqq K\|g\|$ for all $g \in \mathfrak{D}(\pi)$ with $K=\left\|\pi^{*}(A B) f\right\|$ it follows that $\pi^{*}(B) f \in \mathfrak{D}\left(\pi\left(A^{*}\right)^{*}\right)$. Since this is true for all $A \in \mathfrak{A}$ we have 
$\pi^{*}(B) f \in \mathfrak{D}\left(\pi^{*}\right)$. Hence, $\pi^{*}(B) \mathfrak{D}\left(\pi^{*}\right) \subset \mathfrak{D}\left(\pi^{*}\right)$ for all $B \in \mathfrak{U}$ and we have

$$
\begin{aligned}
\left(g, \pi^{*}(A B) f\right) & =\left(\pi\left(A^{*}\right) g, \pi^{*}(B) f\right) \\
& =\left(g, \pi^{*}(A) \pi^{*}(B) f\right)
\end{aligned}
$$

for all $g \in \mathfrak{D}(\pi)$ and $f \in \mathfrak{D}\left(\pi^{*}\right)$. Since $\mathfrak{D}(\pi)$ is dense in $\mathfrak{H}$ we have $\pi^{*}(A B) f$ $=\pi^{*}(A) \pi^{*}(B) f$ for all $A, B \in \mathfrak{A}$ and $f \in \mathfrak{D}\left(\pi^{*}\right)$. Hence, $\pi^{*}$ is a representation of $\mathfrak{A}$.

It is clear that $\pi^{*}$ is an extension of $\pi$ since $\pi\left(A^{*}\right)^{*} \supset \pi(A)$ for all $A \in \mathfrak{A}$.

Finally, we show $\pi^{*}$ is closed. If $\left\{f_{\alpha}: \alpha \in I\right\}$ is a Cauchy set in the induced topology on $\mathfrak{D}\left(\pi^{*}\right)$. Then, it follows from the fact that $\pi\left(A^{*}\right)^{*}$ is a closed operation that if $\lim _{\alpha} f_{\alpha}=f$ then $f \in \mathfrak{D}\left(\pi\left(A^{*}\right)^{*}\right)$ and $\lim _{\alpha} \pi^{*}(A) f_{\alpha}$ $=\pi\left(A^{*}\right)^{*} f$. Hence, $f \in \mathfrak{D}\left(\pi\left(A^{*}\right)^{*}\right)$ for all $A \in \mathfrak{A}$. Hence, $f \in \mathfrak{D}\left(\pi^{*}\right)$ and $\mathfrak{D}\left(\pi^{*}\right)$ is complete and, therefore, $\pi^{*}$ is closed. This completes the proof of the lemma.

Lemma 4.2. Every hermitian extension of $a^{*}$-representation $\pi$ is an hermitian restriction of $\pi^{*}$. (In fact, if $\pi, \pi_{1}$ are hermitian and $\pi_{1} \supset \pi$ then $\pi^{*} \supset \pi_{1}^{*} \supset \pi_{1} \supset \pi$.)

Proof. Suppose $\pi$ is a *-representation of a *-algebra $\mathfrak{A}$ on a Hilbert space $\mathfrak{S}$ and $\pi_{1}$ is an hermitian extension of $\pi$. Since $\pi_{1}\left(A^{*}\right) \supset \pi\left(A^{*}\right)$ for all $A \in \mathfrak{A}$ we have $\pi\left(A^{*}\right)^{*} \supset \pi_{1}\left(A^{*}\right)^{*}$. Since $\pi_{1}$ is a *-representation we have $\pi_{1}\left(A^{*}\right)^{*} \supset \pi_{1}(A)$ for all $A \in \mathfrak{A}$. Hence, we have

$$
\pi\left(A^{*}\right)^{*} \supset \pi_{1}\left(A^{*}\right)^{*} \supset \pi_{1}(A) \supset \pi(A)
$$

for all $A \in \mathfrak{A}$. Hence, $\pi^{*} \supset \pi_{1}^{*} \supset \pi_{1} \supset \pi$. Hence $\pi_{1}$ is an hermitian restriction of $\pi^{*}$ and the proof is complete.

Definition 4.3. We say a *-representation $\pi$ of a *-algebra $\mathfrak{A}$ is maximal if every hermitian extension of $\pi$ coincides with $\pi$.

Definition 4.4. We say a representation $\pi$ of a *algebra is self-adjoint if $\pi$ is hermitian and $\pi=\pi^{*}$. We say $\pi$ is essentially self-adjoint if the closure of $\pi$ is self-adjoint.

It follows from Lemma 4.1 that an hermitian representation $\pi$ is selfadjoint if and only if $\mathfrak{D}(\pi)=\mathfrak{D}\left(\pi^{*}\right)$. A self-adjoint representation is maximal by Lemma 4.2 and, therefore closed.

We note that if $\pi^{*}$ is hermitian then $\pi^{*}$ is self-adjoint. This may be seen as follows. Since $\pi^{*}$ is hermitian we have $\pi^{* *} \supset \pi^{*}$ and since $\pi^{*}$ is an hermitian extension of $\pi$ it follows from Lemma 4.2 that $\pi^{* *} \subset \pi^{*}$. Hence, we have $\pi^{*}=\pi^{* *}$ and $\pi^{*}$ is self-adjoint.

We leave open the problem of the general analysis of $\pi^{* *}$. We remark that we have found a simple example of a closed *-representation such 
that $\pi$ is not self-adjoint but $\pi^{*}$ is self-adjoint and hence $\pi^{* *}$ is a proper extension of $\pi$. In general we do not know whether $\pi^{* *}$ is hermitian.

The next lemma shows that operators in the commutant of a *-representation map $\mathfrak{D}(\pi)$ into $\mathfrak{D}\left(\pi^{*}\right)$.

Lemma 4.5. Suppose $\pi$ is a ${ }^{*}$-representation of $a *$-algebra $\mathfrak{A}$ on a Hilbert space $\mathfrak{H}$. Suppose $C \in \pi(\mathfrak{2})^{\prime}$. Then $C \mathfrak{D}(\pi) \subset \mathfrak{D}\left(\pi^{*}\right)$ and $C \pi(A) f$ $=\pi^{*}(A) C f$ for all $A \in \mathfrak{A}$ and $f \in \mathfrak{D}(\pi)$.

Proof. Suppose $\pi$ is a *-representation of $\mathfrak{A}$ and $C \in \pi(\mathfrak{H})^{\prime}$. Then, for all $A \in \mathfrak{A}$ and $f, g \in \mathfrak{D}(\pi)$ we have

$$
(f, C \pi(A) g)=\left(\pi\left(A^{*}\right) f, C g\right) .
$$

Since $\left|\left(\pi\left(A^{*}\right) f, C g\right)\right| \leqq K\|f\|$ for all $f \in \mathfrak{D}(\pi)$ (with $\left.K=\|C \pi(A) g\|\right)$ it follows that $C g \in \mathfrak{D}\left(\pi\left(A^{*}\right)^{*}\right)$ and

$$
(f, C \pi(A) g)=\left(f, \pi\left(A^{*}\right)^{*} C g\right) .
$$

Since $\mathfrak{D}(\pi)$ is dense in $\mathfrak{H}$ we have $C \pi(A) g=\pi\left(A^{*}\right)^{*} C g$. Since $C g \in \mathfrak{D}\left(\pi\left(A^{*}\right)^{*}\right)$ for all $A \in \mathfrak{A}$ we have $C g \in \mathfrak{D}\left(\pi^{*}\right)$. Hence, $C \mathfrak{D}(\pi) \subset \mathfrak{D}\left(\pi^{*}\right)$ and $C \pi(A) f$ $=\pi^{*}(A) C f$ for all $A \in \mathfrak{A}$ and $f \in \mathfrak{D}(\pi)$. This completes the proof of the lemma.

Lemma 4.6. Suppose $\pi$ is a self-adjoint representation of a*-algebra. Then the commutant $\pi(\mathfrak{A})^{\prime}$ is a von Neumann algebra. Furthermore for each $C \in \pi(\mathfrak{A})^{\prime}$ we have $C \mathfrak{D}(\pi) \subset \mathfrak{D}(\pi)$ and $C \pi(A) f=\pi(A) C f$ for all $A \in \mathfrak{A}$ and $f \in \mathfrak{D}(\pi)$.

Proof. Suppose $\pi$ is a self-adjoint representation of $\mathfrak{A}$. Since $\mathfrak{D}(\pi)$ $=\mathfrak{D}\left(\pi^{*}\right)$ it follows that for each $C \in \pi(\mathfrak{2})^{\prime}$ we have $C \mathfrak{D}(\pi) \subset \mathfrak{D}(\pi)$ and $C \pi(A) f=\pi(A) C f$ for all $A \in \mathfrak{A}$ and $f \in \mathfrak{D}(\pi)$. We show $\pi(\mathfrak{R})^{\prime}$ is a von Neumann algebra. Since $\pi(\mathfrak{A})^{\prime}$ is a symmetric weakly closed linear manifold in $\mathfrak{B}(\mathfrak{H})$ it suffices to show that $C_{1}, C_{2} \in \pi(\mathfrak{H})^{\prime}$ implies the product $C_{1} C_{2} \in \pi(\mathfrak{l})^{\prime}$. But, if $C_{1}, C_{2} \in \pi(\mathfrak{H})^{\prime}$ we have that

$$
C_{1} C_{2} \pi(A) f=C_{2} \pi(A) C_{1} f=\pi(A) C_{1} C_{2} f
$$

for all $A \in \mathfrak{A}$ and $f \in \mathfrak{D}(\pi)$. Hence, $C_{1} C_{2} \in \pi(\mathfrak{Q})^{\prime}$. This completes the proof of the lemma.

We turn next to the problem of reducing subspaces. If $\pi$ is a representation of an algebra $\mathfrak{A}$ on a Hilbert space $\mathfrak{H}$ and $\mathfrak{M}$ is a linear manifold contained in $\mathfrak{D}(\pi)$ we say $\mathfrak{M}$ reduces $\pi$ if $\pi(A) \mathfrak{M} \subset \mathfrak{M}$ for all $A \in \mathfrak{A}$. We denote by $\pi \mid \mathfrak{M}$ the representation $\pi$ restricted to $\mathfrak{M}$. If $\pi$ is a *-representation of a *algebra $\mathfrak{A}$ and $\mathfrak{M}$ is a reducing subspace then the restriction $\pi \mid \mathfrak{M}$ is a $*$-representation.

For a bounded *-representation of a *-algebra $\mathfrak{A}$ for each reducing subspace $\mathfrak{M}$ the hermitian projection $E$ onto the closure of $\mathfrak{M}$ is in the 
commutant $\pi(\mathfrak{l})^{\prime}$. This is not true in general for unbounded *-representation. This may be seen as follows.

Let $\mathfrak{A}$ be the free commutative algebra on one hermitian generator $A_{0} . \mathfrak{A}$ consists of all polynomials in $A_{0}$. Let $\mathfrak{S}=L^{2}(R)$ and let $\mathfrak{D}(\pi)$ $=\left\{f \in \mathfrak{H} ; f\right.$ is a $C^{\infty}$-function and $d^{n} f / d x^{n} \in \mathfrak{H}$ for all $\left.n=1,2, \ldots\right\}$. We define a ${ }^{*}$-representation of $\mathfrak{A}$ on $\mathfrak{H}$ defining

$$
\left(\pi\left(A_{0}\right) f\right)(x)=-i \frac{d f}{d x}(x)
$$

for all $f \in \mathfrak{D}(\pi)$. It is easily shown that $\pi$ is a *-representation (in fact $\pi$ is self-adjoint).

Let $\mathfrak{M}$ be the linear manifold of all $C^{\infty}$-functions which vanish outside the closed interval $[0,1]$. Clearly, $\mathfrak{M}$ is a reducing subspace for $\pi$. The hermitian projection $E$ onto the closure of $\mathfrak{M}$ is given by

$$
\begin{aligned}
(E f)(x) & =f(x) & & x \in[0,1] \\
& =0 & & x \notin[0,1]
\end{aligned}
$$

for all $f \in \mathfrak{H}$. However, $E$ is not in $\pi(\mathfrak{A})^{\prime}$ since for $f, g \in \mathfrak{D}(\pi)$ we have

$$
\begin{aligned}
(E f, & \left.\pi\left(A_{0}\right) g\right)-\left(\pi\left(A_{0}^{*}\right) f, E g\right) \\
& =-i \int_{0}^{1}\left(\overline{f(x)} \frac{d}{d x} g(x)+\left(\frac{d \bar{f}}{d x}\right)(x) g(x)\right) d x \\
& =i(\bar{f}(0) g(0)-\bar{f}(1) g(1)) .
\end{aligned}
$$

Since this expression does not vanish for all $f, g \in \mathfrak{D}(\pi), E \notin \pi(\mathfrak{H})^{\prime}$.

We say that a reducing subspace $\mathfrak{M}$ is self-adjoint if $\pi \mid \mathfrak{M}$ is a selfadjoint representation. The next theorem shows that for self-adjoint representations there is a one-to-one correspondence between selfadjoint reducing subspaces and hermitian projections in the commutant.

Theorem 4.7. Suppose $\pi$ is a self-adjoint representation of a *algebra $\mathfrak{U}$ on a Hilbert space $\mathfrak{H}$. Suppose $E \in \pi(\mathfrak{l})^{\prime}$ is an hermitian projection. Let $\mathfrak{M}=E \mathfrak{D}(\pi)$. Then $\mathfrak{M}$ reduces $\pi$ and the restriction $\pi \mid \mathfrak{M}$ is self-adjoint.

Conversely suppose $\mathfrak{M} \subset \mathfrak{D}(\pi)$ is a reducing subspace for $\pi$ and $\pi \mid \mathfrak{M}$ is self-adjoint. Then, the hermitian projection $E_{\mathfrak{M}}$ onto the closure of $\mathfrak{M}$ is in the commutant $\pi(\mathfrak{Q})^{\prime}$.

Hence, for self-adjoint representations there is a one-to-one correspondence between hermitian projections in $\pi(\mathfrak{H})^{\prime}$ and self-adjoint reducing subspaces.

Proof. Suppose $\pi$ is a self-adjoint representation of a ${ }^{*}$-algebra $\mathfrak{A}$. Suppose $E \in \pi(\mathfrak{A})^{\prime}$ is an hermitian projection. Let $\mathfrak{M}=E \mathfrak{D}(\pi)$. From Lemma 4.6 it follows that $E \mathfrak{D}(\pi) \subset \mathfrak{D}(\pi)$ and $\pi(A) E f=E \pi(A) E f$ for all $A \in \mathfrak{A}$ and all $f \in \mathfrak{D}(\pi)$. Hence, $\mathfrak{M} \subset \mathfrak{D}(\pi)$ and $\mathfrak{M}$ reduces $\pi$. 
We show $\pi \mid \mathfrak{M}$ is self-adjoint. Suppose $f \in \overline{\mathfrak{M}}$ and $f \in \mathfrak{D}\left((\pi / \mathfrak{M})^{*}\right)$. Then $f \in \mathfrak{D}\left((\pi(A) / \mathfrak{M})^{*}\right)$ for all $A \in \mathfrak{A}$. Hence, for each $A \in \mathfrak{U}$ there is a constant $K_{A}$ depending only on $A$ and $f$ such that $|(f, \pi(A) g)| \leqq K_{A}\|g\|$ for all $g \in \mathfrak{M}$. Since $E f=f$ we have for all $g \in \mathfrak{D}(\pi)$

$$
\begin{aligned}
|(f, \pi(A) g)| & =|(E f, \pi(A) g)|=|(f, E \pi(A) g)| \\
& =|(f, \pi(A) E g)| \leqq K_{A}\|E g\| \leqq K_{A}\|g\| .
\end{aligned}
$$

Hence, $f \in \mathfrak{D}\left(\pi(A)^{*}\right)$ for all $A \in \mathfrak{A}$. Hence, $f \in \mathfrak{D}\left(\pi^{*}\right)=\mathfrak{D}(\pi)$. Since $E f=f$ we have $f \in E \mathfrak{D}(\pi)=\mathfrak{M}$. Hence, $f \in \mathfrak{D}\left((\pi / \mathfrak{M})^{*}\right)$ implies $f \in \mathfrak{M}$ and, therefore, $\pi / \mathfrak{M}$ is self-adjoint.

Conversely, suppose $\mathfrak{M}$ reduces $\pi$ and $\pi / \mathfrak{M}$ is self-adjoint. Let $E$ be the hermitian projection onto the closure of $\mathfrak{M}$. Clearly, we have $E \mathfrak{D}(\pi) \supset \mathfrak{M}$. We show that $\mathfrak{M} \supset E \mathfrak{D}(\pi)$. Suppose $f \in E \mathfrak{D}(\pi)$. Then $f=E f_{1}$ with $f_{1} \in \mathfrak{D}(\pi)$. We have for all $g \in \mathfrak{M}$

$$
\begin{aligned}
(f, \pi(A) g) & =\left(E f_{1}, \pi(A) g\right) \\
& =\left(f_{1}, E \pi(A) g\right) \\
& =\left(f_{1}, \pi(A) g\right) .
\end{aligned}
$$

Since $f_{1} \in \mathfrak{D}(\pi) \subset \mathfrak{D}\left(\pi^{*}\right)$ we have that $f_{1} \in \mathfrak{D}\left(\pi(A)^{*}\right)$ for all $A \in \mathfrak{A}$. Hence, for each $A \in \mathfrak{A}$ there is a constant $K_{A}$ depending only on $A$ and $f_{1} \in \mathfrak{D}\left(\pi(A)^{*}\right)\left(K_{A}=\left\|\pi(A)^{*} f_{1}\right\|\right)$ such that $\left|\left(f_{1}, \pi(A) g\right)\right| \leqq K_{A}\|g\|$ for all $g \in \mathfrak{M} \subset \mathfrak{D}(\pi)$. Then, from the above equation it follows that

$$
|(f, \pi(A) g)|=\left|\left(f_{1}, \pi(A) g\right)\right| \leqq K_{A}\|g\|
$$

for all $g \in \mathfrak{M}$. Hence, $f \in \mathfrak{D}\left((\pi(A) \mid \mathfrak{M})^{*}\right)$ for all $A \in \mathfrak{A}$ and, therefore, $f \in \mathfrak{D}\left((\pi \mid \mathfrak{M})^{*}\right)$. Since $\pi \mid \mathfrak{M}$ is self-adjoint we have $f \in \mathfrak{M}$. Hence, $E \mathfrak{D}(\pi) \subset \mathfrak{M}$ and, therefore, $E \mathfrak{D}(\pi)=\mathfrak{M}$.

Since $E \mathfrak{D}(\pi)=\mathfrak{M} \subset \mathfrak{D}(\pi)$ it follows that for $f, g \in \mathfrak{D}(\pi)$ and $A \in \mathfrak{A}$ we have

and

$$
\left(\pi\left(A^{*}\right) f, E g\right)=(f, \pi(A) E g)=(f, E \pi(A) E g)
$$

$$
\begin{aligned}
(f, E \pi(A) g) & =(E f, \pi(A) g)=\left(\pi\left(A^{*}\right) E f, g\right) \\
& =\left(E \pi\left(A^{*}\right) E f, g\right)=\left(\pi\left(A^{*}\right) E f, E g\right) \\
& =(E f, \pi(A) E g)=(f, E \pi(A) E g)
\end{aligned}
$$

Hence, $\left(\pi\left(A^{*}\right) f, E g\right)=(f, E \pi(A) g)$ for all $A \in \mathfrak{U}$ and $f, g \in \mathfrak{D}(\pi)$. Hence, $E \in \pi(\mathfrak{A})^{\prime}$. This completes the proof of the theorem.

Remarks. We note that in the proof of the second part of the above theorem we did not use the fact that $\pi$ was self-adjoint. Hence, we have the slightly stronger statement for the second part of Theorem 4.7. If $\pi$ 
is a *-representation of a *-algebra $\mathfrak{A}$ on a Hilbert space $\mathfrak{S}$ and $\mathfrak{M} \subset \mathfrak{D}(\pi)$ is a reducing subspace for $\pi$ such that $\pi \mid \mathfrak{M}$ is essentially self-adjoint then $E_{\mathfrak{M}} \in \pi(\mathfrak{O})^{\prime}$ where $E_{\mathfrak{M}}$ is the hermitian projection onto the closure of $\mathfrak{M}$.

Using some of the ideas of Nelson [7] we define the notion of domination as follows.

Definition 4.8. Suppose $\pi$ is a $*$-representation of a *algebra $\mathfrak{A}$ on a Hilbert space $\mathfrak{G}$. Suppose $S \subset \mathfrak{A}$ is a symmetric linear subset of $\mathfrak{U}$. We say $\pi(S)$ dominates $\pi(\mathfrak{l})$ if for every $A \in \mathfrak{A}$ there is a $B \in S$ such that $\|\pi(B) f\| \geqq\|\pi(A) f\|$ for all $f \in \mathfrak{D}(\pi)$. We say $S$ dominates $\mathfrak{U}$ if $\pi(S)$ dominates $\pi(\mathfrak{Q})$ for every ${ }^{*}$-representation $\pi$ of $\mathfrak{A}$.

A very simple example of an algebra and a dominating subalgebra is the following. Let $\mathfrak{A}$ be the free commutative algebra on two hermitian generators $A$ and $B$. $\mathfrak{A}$ consists of all polynomials in the commuting elements $A$ and $B$. Let $\mathfrak{U}_{1}$ be the *-subalgebra of $\mathfrak{A}$ consisting of all polynomials in $H=A^{2}+B^{2}$. It is simple matter to show that $\pi\left(\mathfrak{A}_{1}\right)$ dominates $\pi(\mathfrak{H})$ for every ${ }^{*}$-representation $\pi$ of $\mathfrak{A}$.

Lemma 4.9. Suppose $\pi$ is $a^{*}$-representation of $a *$-algebra $\mathfrak{A}$ and suppose $\mathfrak{U}_{1}$ is a *-subalgebra such that $\pi\left(\mathfrak{A}_{1}\right)$ dominates $\pi(\mathfrak{A})$. Let $\pi_{1}$ be *-representation of $\mathfrak{Q}_{1}$ defined by $\pi_{1}(A)=\pi(A)$ for all $A \in \mathfrak{A}_{1}$ and $\mathfrak{D}\left(\pi_{1}\right)$ $=\mathfrak{D}(\pi)$ (i.e. $\pi_{1}=\pi \mid \mathfrak{U}_{1}$ ) then, if $\pi_{1}$ is self-adjoint then $\pi$ is self-adjoint.

Proof. Let $\pi$ and $\pi_{1}$ be *-representations of $\mathfrak{Q}$ and $\mathfrak{U}_{1}$ as described in the lemma. Since $\pi_{1}=\pi_{1}^{*}$ we have $\mathfrak{D}\left(\pi_{1}^{*}\right)=\mathfrak{D}\left(\pi_{1}\right)=\mathfrak{D}(\pi)$. Since $\mathfrak{D}\left(\pi_{1}^{*}\right) \supset \mathfrak{D}\left(\pi^{*}\right)$ we have $\mathfrak{D}\left(\pi^{*}\right) \subset \mathfrak{D}(\pi)$. Since $\mathfrak{D}\left(\pi^{*}\right) \supset \mathfrak{D}(\pi)$ we have $\mathfrak{D}(\pi)$ $=\mathfrak{D}\left(\pi^{*}\right)$ and $\pi=\pi^{*}$. This completes the proof of the lemma.

We remark that the converse of this lemma is false. In Example 3 of the next section we describe a self-adjoint representation of the free commutative algebra on two hermitian generators $A$ and $B$ such that the *-subalgebra generated by $\pi\left(A^{2}+B^{2}\right)$ is not self-adjoint.

\section{Some Examples of Unbounded Self-Adjoint Representations}

In this section we examine three examples of self-adjoint representations of *-algebras. The first two examples are well known. The first example is a representation of a commutative *-algebra by an algebra of functions on a measure space. The second example is the "Schrodinger" representation of the commutation relations. The third example is a modification of an example constructed by Nelson in [7]. Nelson constructed an example of two essentially self-adjoint operators $A$ and $B$ defined on a common dense invariant domain $\mathfrak{D}$, such that $A B f=B A f$ for all $f \in \mathfrak{D}$, but the spectral projections of $\bar{A}$ fail to commute with the spectral projections of $\bar{B}$. By modifying Nelson's example we construct 
an irreducible self-adjoint representation of the free commutative algebra or on two hermitian generators on an infinite dimensional Hilbert space.

Example 1. For the first example we consider the free commutative algebra $\mathfrak{A}$ on $n$-hermitian generators $A_{1}, \ldots, A_{n}$. $\mathfrak{U}$ consists of all polynomials

$$
P=\alpha[m] \sum_{[m]} \alpha([m]) A^{[m]} \quad \text { where } \quad A^{[m]}=A_{1}^{m_{1}} A_{2}^{m_{2}} \ldots A_{n}^{m_{n}}
$$

and the $\alpha([m])$ are complex numbers with $\alpha([m]) \neq 0$ for only a finite number of $[m]$. Let $\mu$ be a regular Borel measure on $R^{n}$ and let $\mathfrak{H} \quad L^{2}\left(R^{n}, \mu\right)$ be the Hilbert space of all $\mu$-measurable square integrable functions on $R^{n}$. We define a *-representation $\pi$ on $\mathfrak{H}$ by the relations,

$$
(\pi(P) f)\left(x_{1} \ldots x_{n}\right)=P\left(x_{1} \ldots x_{n}\right) f\left(x_{1} \ldots x_{n}\right)
$$

for all $P=P\left(A_{1}, \ldots A_{n}\right) \in \mathfrak{U}$. We take the domain of $\pi$ to be

$$
\mathfrak{D}(\pi)=\left\{f \in \mathfrak{H} ; \int|P(x) f(x)|^{2} d \mu(x)<\infty \quad \text { for all } \quad P \in \mathfrak{A}\right\} .
$$

Clearly, $\pi$ is a *-representation of $\mathfrak{A}$.

We will show that $\pi$ is a self-adjoint representation of $\mathfrak{A}$. First we will show $\pi\left(P^{*}\right)^{*}=\overline{\pi(P)}$ for all $P \in \mathfrak{A}$. To prove this it is enough to show $\overline{\pi(P)} \supset \pi\left(P^{*}\right)^{*}$ since $\pi\left(P^{*}\right)^{*} \supset \overline{\pi(P)}$ for all $P \in \mathfrak{A}$. Suppose $f \in \mathfrak{D}\left(\pi\left(P^{*}\right)^{*}\right)$ and $\pi\left(P^{*}\right)^{*} f=g$. Since

$$
\int \overline{(P(x) f(x)-g(x))} h(x) d \mu(x)=0
$$

for all $h \in \mathfrak{D}(\pi)$ and in particular for all square integrable functions $h$ of compact support it follows that $g(x)=P(x) f(x) \mu$-almost everywhere. Let

Then, we have

$$
\begin{aligned}
f_{n}(x) & =f(x) & & \text { for } & & |x| \leqq n \\
& =0 & & \text { for } & & |x|>n .
\end{aligned}
$$

$$
\begin{aligned}
\left\|f-f_{n}\right\|^{2} & =\int_{|x|>n}|f(x)|^{2} d \mu(x) \rightarrow 0, \\
\left\|\pi(P) f_{n}-g\right\|^{2} & =\int_{|x|>n}|g(x)|^{2} d \mu(x) \rightarrow 0
\end{aligned}
$$

as $n \rightarrow \infty$ by the Lebesque dominated convergence theorem. Hence, $f \in \mathfrak{D} \overline{(\pi(P))}$ and $\overline{\pi(P)} f=g$. Hence, we have $\pi\left(P^{*}\right)^{*}=\overline{\pi(P)}$ for all $P \in \mathfrak{A}$ and, therefore,

$$
\mathfrak{D}\left(\pi^{*}\right)=\bigcap_{P \in \mathfrak{A}} \mathfrak{D}\left(\pi(P)^{*}\right)=\bigcap_{P \in \mathfrak{A}} \mathfrak{D} \overline{\left(\pi\left(P^{*}\right)\right)}=\mathfrak{D}(\pi) .
$$

Hence, $\pi$ is self-adjoint.

Example 2. For the second example we take the Schrodinger representation of the canonical commutation relations for a finite number of 
degrees of freedom. Let $\mathfrak{A}$ be the free non-commutative algebra of all polynomials in the hermitian generators $\left\{q_{i}, p_{i} ; i=1, \ldots, s\right\}$ modulo the two-sided ${ }^{*}$-ideal generated by the relations

$$
\begin{aligned}
& {\left[q_{i}, p_{j}\right]=q_{i} p_{j}-p_{i} q_{i}=i \delta_{i j} I,} \\
& {\left[q_{i}, q_{j}\right]=\left[p_{i}, p_{j}\right]=0}
\end{aligned}
$$

for $i, j=1, \ldots, s$.

We define a *-representation $\pi$ on $\mathfrak{G}=L^{2}\left(R^{s}\right)$ by defining

$$
\begin{aligned}
& \left(\pi\left(q_{i}\right) f\right)(x)=x_{i} f(x) . \\
& \left(\pi\left(p_{i} f\right)\right)(x)=-i\left(\frac{\partial}{\partial x_{i}} f\right)(x) .
\end{aligned}
$$

We define $\mathfrak{D}(\pi)$ to be Schwartz's space $\mathscr{S}\left(R^{s}\right)$ of all complex infinitely differentiable functions which together with their derivatives decrease faster than any polynomial in $x_{1}, \ldots, x_{s}$.

Clearly $\pi$ is a ${ }^{*}$-representation of $\mathfrak{A}$. It follows from [5, Part II] or [13] that the induced topology on $\mathfrak{D}(\pi)=\mathscr{S}\left(R^{s}\right)$ is equivalent to the Schwartz space topology on $\mathscr{S}\left(R^{s}\right)$. Let $H=\frac{1}{2} \sum_{i=1}^{s} p_{i}^{2}+q_{i}^{2}$ and let $\mathfrak{U}_{1}$ be the *-subalgebra of $\mathfrak{A}$ consisting of all polynomials in $H$. It is well known that $\pi\left(H^{n}\right)=\left(\frac{1}{2}\left(|x|^{2}-\Delta\right)\right)^{n} / \mathscr{S}\left(R^{s}\right)$ is essentially self-adjoint for all $n=1,2, \ldots$ It is shown in [5, Part II] or [13] that a function $f \in \mathfrak{H}=L^{2}\left(R^{s}\right)$ is in $\mathscr{S}\left(R^{s}\right)$ if and only if $f \in \mathfrak{D}\left(\overline{\left.\pi\left(H^{n}\right)\right)}\right.$ for all $n=1,2, \ldots$ and furthermore the topology on $\mathscr{S}\left(R^{s}\right)$ defined by the semi-norms $\|f\|_{n}=\left\|\pi\left(H^{n}\right) f\right\|$ is equivalent to the Schwartz space topology on $\mathscr{S}\left(R^{s}\right)$.

It follows from this remark that $\pi\left(\mathfrak{A}_{1}\right)$ dominates $\pi(\mathfrak{H})$ (in fact one can easily check that $\mathfrak{A}_{1}$ dominates $\left.\mathfrak{A}\right)$. Since $\mathscr{S}\left(R^{s}\right)$ is complete it follows that $\pi$ and $\pi_{1}=\pi \mid \mathfrak{A}_{1}$ are closed *-representations of $\mathfrak{U}$ and $\mathfrak{U}_{1}$. Since $\overline{\pi_{1}\left(H^{n}\right)}=\overline{\pi\left(H^{n}\right)}$ is self-adjoint for all $n=1,2, \ldots$ it follows that

$$
\mathfrak{D}\left(\pi_{1}^{*}\right)=\bigcap_{n=1}^{\infty} \mathfrak{D}\left(\pi_{1}\left(H^{n}\right)^{*}\right)=\bigcap_{n=1}^{\infty} \mathfrak{D}\left(\overline{\pi_{1}\left(H^{n}\right)}\right)=\mathfrak{D}\left(\pi_{1}\right) .
$$

Hence, $\pi_{1}$ is self-adjoint and from Lemma 4.9 it follows that $\pi$ is selfadjoint.

We show $\pi$ is irreducible. It is well known that $\pi\left(q_{i}\right)=x_{i} \mid \mathscr{S}\left(R^{s}\right)$ and $\pi\left(p_{i}\right)=-i\left(\partial / \partial x_{i}\right) \mid \mathscr{S}\left(R^{s}\right)$ are essentially self-adjoint for $i=1, \ldots, s$. If $C \in \pi(\mathfrak{Q})^{\prime}, C$ must commute with $\pi\left(q_{i}\right)$ and $\pi\left(p_{i}\right)$ for $i=1, \ldots, s$. Since these operators are self-adjoint $C$ must commute with their spectral projections and therefore, $C$ must commute with the unitary operators. $U(k)$ and 
$V(y)$ for all $k, y \in R^{s}$, where

$$
\begin{aligned}
& \left.(U(k) f)(x)=\left(\exp \overline{\left(i \pi\left(\sum_{i=1}^{s} k_{i} q_{i}\right)\right.}\right) f\right)(x)=e^{i k \cdot x} f(x), \\
& \left.(V(y) f)(x)=\left(\exp \overline{\left(i \pi\left(\sum_{i=1}^{s} y_{i} P_{i}\right)\right.}\right) f\right)(x)=f(x+y) .
\end{aligned}
$$

It is well known that these operators (defining the Weyl-Schrodinger representation of the commutation relations) are irreducible (see e.g. [17]). Hence, $C \in \pi(\mathfrak{U})^{\prime}$ implies $C=\lambda I$. Hence, $\pi$ is irreducible.

We note that although $\pi$ is irreducible not every vector $f \in \mathfrak{D}(\pi)$ is cyclic. If $f \in \mathfrak{D}(\pi)$ has compact support then every $g \in\{\pi(\mathfrak{U}) f\}$ has support in the same compact set and, therefore, $f$ is certainly not cyclic for $\pi$.

Example 3. By modifying an example of Nelson [7] we construct an irreducible representation on an infinite dimensional Hilbert space of the free commutative algebra on two hermitian generators. Let $\mathfrak{A}$ be the free commutative algebra on two hermitian generators $A$ and $B$. $\mathfrak{U}$ is *-isomorphic to the *-algebra of all complex polynomials in two real variables. Let $\mathfrak{H}=L^{2}(V)$ be the Hilbert space of all Lebesque measurable square integrable functions in the square $V=\{(x, y) ;|x| \leqq \pi,|y| \leqq \pi\}$. Let $\mathfrak{D}(\pi)$ be all $C^{\infty}$-functions (infinitely differentiable) on $V$ satisfying the boundary conditions.

$$
\begin{aligned}
& \left(\frac{\partial^{n} f}{\partial x^{n}}\right)(-\pi, y)=e^{i y}\left(\frac{\partial^{n} f}{\partial x^{n}}\right)(\pi, y) \text { for all }|y| \leqq \pi \\
& \left(\frac{\partial^{n} f}{\partial y^{n}}\right)(x,-\pi)=\left(\frac{\partial^{n} f}{\partial y^{n}}\right)(x, \pi) \text { for all }|x| \leqq \pi
\end{aligned}
$$

for all $n=0,1,2, \ldots$ We define

$$
\begin{aligned}
& (\pi(A) f)(x, y)=-i\left(\frac{\partial f}{\partial x}\right)(x, y), \\
& (\pi(B) f)(x, y)=-i\left(\frac{\partial f}{\partial y}\right)(x, y)
\end{aligned}
$$

for all $f \in \mathfrak{D}(\pi)$. Clearly $\pi(A) \mathfrak{D}(\pi) \subset \mathfrak{D}(\pi), \pi(B) \mathfrak{D}(\pi) \subset \mathfrak{D}(\pi)$ and $\pi(A) \pi(B) f$ $=\pi(B) \pi(A) f$ for all $f \in \mathfrak{D}(\pi)$. We define $\pi(P)$ for all $P=P(A, B) \in \mathfrak{U}$ polynomials in $A$ and $B$ by the relation $\pi(P) f=P(\pi(A), \pi(B)) f$ for all $f \in \mathfrak{D}(\pi)$. Clearly $\pi$ is a *-representation of $\mathfrak{U}$.

Lemma 5.1. $\pi\left(A^{n}\right)$ and $\pi\left(B^{n}\right)$ are essentially self-adjoint in $\mathfrak{D}(\pi)$ for all $n=1,2, \ldots$. 
Proof. Consider the function $f(x, y)$ of the form

$$
f(x, y)=\left[\exp \left(\frac{-i x y}{2 \pi}+i m x\right) h(y)\right]
$$

where $m$ is an integer and $h(y)$ is a $C^{\infty}$-function with suppoert strictly inside the interval $(-\pi, \pi)$ (i.e., there is a $\delta>0$ depending on $h$ such that $h(y)=0$ for $|y|<\pi-\delta)$, one can easily verify that $f \in \mathfrak{D}(\pi)$. We claim that $f$ is an analytic vector for $\pi\left(A^{n}\right)$. In fact, we have

$$
\begin{aligned}
(\pi(A) f)(x, y) & =(m-y / 2 \pi) f(x, y), \\
\left(\pi\left(A^{n}\right)^{k} f\right)(x, y) & =(m-y / 2 \pi)^{n k} f(x, y), \\
\|\left(\pi\left(A^{n}\right)^{k} f\right) \mid & \leqq\left\|m\left|+\frac{1}{2}\right|^{n k}\right\| f \| .
\end{aligned}
$$

Hence, we have

$$
\begin{gathered}
\sum_{k=0}^{\infty} \frac{\left\|\pi\left(A^{n}\right)^{k} f\right\|}{k^{\prime} !} s^{k} \leqq\|f\| \sum_{k=0}^{\infty} \frac{\left\|m\left|+\frac{1}{2}\right|^{n k} s^{k}\right\|}{k^{\prime} !} \\
=\|f\| \exp \left(s\left(|m|+\frac{1}{2}\right)^{n}\right)<\infty .
\end{gathered}
$$

Hence, $f$ is an analytic vector of $\pi\left(A^{n}\right)$.

Since the linear span of functions $f$ of the form (a) is dense in $\mathfrak{H}$ it follows that $\mathfrak{D}(\pi)$ contains a dense set of analytic vectors for $\pi\left(A^{n}\right)$. Hence by a theorem of Nelson [7, Lemma 5.1$], \pi\left(A^{n}\right)$ is essentially self-adjoint.

Next consider the function $g(x, y)$ of the form

$$
g(x, y)=k(x) e^{i m y}
$$

where $m$ is an integer and $k(x)$ is a $C^{\infty}$-function which support strictly inside the interval $(-\pi, \pi)$. We claim $g$ is an analytic vector for $\pi\left(B^{n}\right)$. In fact, we have

$$
\begin{gathered}
(\pi(B) g)(x, y)=m g(x, y), \\
\left(\pi\left(B^{n}\right) g\right)(x, y)=m^{n} g(x, y) .
\end{gathered}
$$

Hence, $g$ is an analytic vector for $\pi\left(B^{n}\right)$. Since the linear span of vectors $g$ of the form (b) is dense in $\mathfrak{H}$ it follows that $\mathfrak{D}(\pi)$ contains a dense set of analytic vectors for $\pi\left(B^{n}\right)$. Hence, $\pi\left(B^{n}\right)$ is essentially self-adjoint.

Lemma 5.2. $\pi^{*}$ is hermitian and therefore $\pi^{*}$ is self-adjoint.

Proof. Suppose $\pi^{*}$ is the hermitian adjoint of $\pi$ and $f, g \in \mathfrak{D}\left(\pi^{*}\right)$. We show that $\left(f, \pi^{*}\left(A^{n} B^{m}\right) g\right)=\left(\pi^{*}\left(A^{n} B^{m}\right) f, g\right)$ for all $n, m=0,1,2, \ldots$. Since $\overline{\pi\left(A^{n}\right)}$ and $\overline{\pi\left(B^{m}\right)}$ are self-adjoint by Lemma 5.1, we have $\pi^{*}\left(A^{n}\right) f=\overline{\pi\left(A^{n}\right)} f$ and $\pi^{*}\left(B^{m}\right) f=\overline{\pi\left(B^{m}\right)} f$ for all $f \in \mathfrak{D}\left(\pi^{*}\right)$. By Lemma 4.1 we have that $\pi^{*}$ is a representation of $\mathfrak{A}$ and $\pi^{*}(P) \mathfrak{D}\left(\pi^{*}\right) \subset \mathfrak{D}\left(\pi^{*}\right)$ for all $P \in \mathfrak{A}$. Hence 
we have for $f, g \in \mathfrak{D}\left(\pi^{*}\right)$

$$
\begin{aligned}
\left(f, \pi^{*}\left(A^{n} B^{m}\right) g\right) & =\left(f, \pi^{*}\left(A^{n}\right) \pi^{*}\left(B^{m}\right) g\right) \\
& =\left(f, \overline{\pi\left(A^{n}\right)} \overline{\pi\left(B^{m}\right)} g\right) \\
& =\left(\pi^{*}\left(A^{n}\right) f, \overline{\pi\left(B^{m}\right)} g\right) \\
& =\left(\pi^{*}\left(B^{m}\right) \pi^{*}\left(A^{n}\right) f, g\right) \\
& =\left(\pi^{*}\left(B^{m} A^{n}\right) f, g\right)=\left(\pi^{*}\left(A^{n} B^{m}\right) f, g\right) .
\end{aligned}
$$

It follows that for $P \in \mathfrak{A}$ with $P=\sum_{n m} \alpha(n, m) A^{n} B^{m}$ and $f, g \in \mathfrak{D}\left(\pi^{*}\right)$ we have

$$
\begin{aligned}
\left(f, \pi^{*}(P) g\right) & =\sum_{n m} \alpha(n, m)\left(f, \pi^{*}\left(A^{n} B^{m}\right) g\right) \\
& =\sum_{n m} \alpha(n, m)\left(\pi^{*}\left(A^{n} B^{m}\right) f, g\right) \\
& =\left(\pi^{*}\left(P^{*}\right) f, g\right) .
\end{aligned}
$$

Hence, $\pi^{*}$ is hermitian. By the remark after Definition 4.4 it follows that $\pi^{*}$ is self-adjoint.

We remark that a closer analysis shows that $\pi$ itself is already selfadjoint.

Lemma 5.3. Let $U(\alpha)=\exp (i \alpha \overline{\pi(A))}$ and $V(\beta)=\exp \overline{(i \beta \pi}(B))$ for all real $\alpha, \beta$. Then $\pi(\mathfrak{H})^{\prime}=\{U(\alpha), V(\beta),-\infty<\alpha, \beta<\infty\}^{\prime}$.

Proof. Suppose $C$ is bounded operator on $\mathfrak{H}$ which commutes with $U(\alpha)$ and $V(\beta)$ for all real $\alpha$ and $\beta$. Then for $f, g \in \mathfrak{D}(\pi)$ we have

$$
\begin{aligned}
(f, C \pi(A) g) & =\lim _{\alpha \rightarrow 0}(i \alpha)^{-1}(f, C(U(\alpha)-I) g) \\
& =\lim _{\alpha \rightarrow 0}\left((-i \alpha)^{-1}(U(-\alpha)-I) f, C g\right) \\
& =(\pi(A) f, C g)
\end{aligned}
$$

since by Stone's theorem $(i \alpha)^{-1}(U(\alpha)-I) f \rightarrow \pi(A) f$ as $\alpha \rightarrow 0$ for $f \in \mathfrak{D}(\pi)$ $C \mathfrak{D}(\overline{\pi(A)})$. Similarly one finds $(f, C \pi(B) g)=(\pi(B) f, C g)$ for all $f, g \in \mathfrak{D}(\pi)$. By induction we have $\left(f, C \pi\left(A^{n} B^{m}\right) g\right)=\left(\pi\left(A^{n} B^{m}\right) f, C, g\right)$ for all $f, g \in \mathfrak{D}(\pi)$ and $n, m=0,1,2, \ldots$. Hence, $(f, C \pi(P) g)=\left(\pi\left(P^{*}\right) f, C, g\right)$ for all $P \in \mathfrak{U}$ and $f, g \in \mathfrak{D}(\pi)$. Therefore $C \in \pi(\mathfrak{Q})^{\prime}$.

Conservely suppose $C \in \pi(\mathfrak{A})^{\prime}$. Then we have $(f, C \pi(A) g)=(\pi(A) f, C g)$ for all $f, g \in \mathfrak{D}(\pi)$. Since $\pi(A)$ is essentially self-adjoint it follows that $C$ commutes with the spectral projections of $\overline{\pi(A)}$ (see Section I) and hence $C$ commutes with

$$
U(\alpha)=\int e^{i \lambda \alpha} d E(\lambda) \text { with } \pi(A)=\int \lambda d E(\lambda)
$$

and similarly $C$ commutes with $V(\beta)$ for all $\beta$. This completes the proof of the lemma. 
Lemma 5.4. $\pi(\mathfrak{U})^{\prime}=\{\lambda I\}$.

Proof. Let $R$ be the von Neumann algebra generated by $\{U(\alpha), V(\beta)$; $<\infty+\alpha, \beta<+\infty$ \}. By Lemma 5.4 we have $\pi(\mathfrak{Q})^{\prime}=R^{\prime}$.

One can easily check with the aid of Stone's theorem that for $0 \leqq \alpha \leqq 2 \pi$ and $0 \leqq \beta \leqq 2 \pi$, we have for all $f \in \mathfrak{H}=L^{2}(V)$

$$
\begin{aligned}
(U(\alpha) f)(x, y) & =f(x+\alpha, y)-\pi \leqq x<\pi-\alpha \\
& =e^{-i y} f(x+\alpha-2 \pi, y) \pi-\alpha \leqq x \leqq \pi, \\
(U(-\alpha) f)(x, y) & =f(x-\alpha, y) \alpha-\pi \leqq x \leqq \pi \\
& =e^{i y} f(x-\alpha+2 \pi, y)-\pi \leqq x<\alpha-\pi
\end{aligned}
$$

for all $-\pi \leqq y \leqq \pi$, and

$$
\begin{aligned}
(V(\beta) f)(x, y) & =f(x, y+\beta) & & -\pi \leqq y<\pi-\beta \\
& =f(x, y+\beta-2 \pi) & & \pi-\beta \leqq y \leqq \pi, \\
(V(-\beta) f)(x, y-\beta) & =f(x, y-\beta) & & \beta-\pi \leqq y \leqq \pi \\
& =f(x, y-\beta+2 \pi) & & -\pi \leqq x<\beta-\pi
\end{aligned}
$$

for all $-\pi \leqq x \leqq \pi$. Let $E(\lambda)(-\pi \leqq \lambda \leqq \pi)$ be the projection defined by

$$
\begin{array}{rlrl}
(E(\lambda) f)(x, y) & =((I-V(\pi) U(\pi-\lambda) V(-\pi) U(\lambda-\pi)) f)(x, y) \\
& =f(x, y) & & -\pi \leqq x \leqq \lambda \\
& =0 & & \lambda \leqq x \leqq \pi .
\end{array}
$$

We have $E(\lambda) \in R$ for all $|\lambda| \leqq \pi$, since $U(\alpha), V(\beta) \in R$ for all $\alpha$ and $\beta$. Let

$$
S_{1}=\int e^{i \lambda} d E(\lambda) .
$$

We have $S_{1} \in R$ and for all $f \in \mathfrak{H}$

$$
\left(S_{1} f\right)(x, y)=e^{i x} f(x, y) .
$$

Let $S_{2}=U(-2 \pi) \in R$. We have for all $f \in \mathfrak{H}$

$$
\left(S_{2} f\right)(x, y)=e^{i y} f(x, y) \text {. }
$$

Let $R_{1}$ be the von Neumann algebra generated by $S_{1}$ and $S_{2} . R_{1}$ is a commutative von Neumann algebra and the vector $f(x, y)=1 \in \mathfrak{H}$ is cyclic for $R_{1}$ since the linear span of the functions $\left\{e^{i n x} e^{i m x} ; n, m=0 \pm 1, \pm 2, \ldots\right\}$ is dense in $\mathfrak{H}$. Since $R_{1}$ is commutative and has a cyclic vector $R_{1}$ is a maximal commutative von Neumann algebra (see [10, Lemma 1.2]) i.e. $R_{1}=R_{1}^{\prime}$. Since $R_{1} \subset R$ we have $R^{\prime} \subset R_{1}^{\prime}=R_{1}$. Hence, every operator $C \in R^{\prime} \subset R_{1}$ is of the form

$$
(C f)(x, y)=C(x, y) f(x, y)
$$

for all $f \in \mathfrak{H}$, with $\subset(x, y)$ a measurable essentially bounded function. 
Now suppose $C \in R^{\prime}$. We show $C=\lambda I$. Since $C \in R^{\prime} C R_{1}$, we have

$$
(C f)(x, y)=C(x, y) f(x, y)
$$

for all $f \in \mathfrak{H}$. A straight-forward computation shows that

$$
\begin{aligned}
& (U(\alpha) C U(-\alpha) f)(x, y)=C([x+\alpha], y) f(x, y), \\
& (V(\beta) C V(-\beta) f)(x, y)=C(x,[y+\beta]) f(x, y)
\end{aligned}
$$

for all $f \in \mathfrak{H}$ and all $\alpha$ and $\beta$, where $[x+\alpha]=x+\alpha$ modulo $2 \pi$. Since $U(\alpha) C U(-\alpha)=C$ and $V(\beta) C V(-\beta)=C$ for all $\alpha$ and $\beta$ it follows that $C(x, y)$ is constant almost everywhere. Hence $C=\lambda I$ and $\pi(\mathfrak{U})^{\prime}=\{\lambda I\}$.

Theorem 5.5. $\pi^{*}$ is an irreducible self-adjoint representation of $\mathfrak{A}$ on an infinite dimensional Hilbert space.

Proof. By Lemma $5.1 \pi^{*}$ is self-adjoint and by Lemma $5.4 \pi(\mathfrak{A})^{\prime}$ $=\{\lambda I\}$. Since $\pi^{*} \supset \pi$, it follows $\pi^{*}(\mathfrak{U})^{\prime} \subset \pi(\mathfrak{U})^{\prime}$. Hence, $\pi^{*}(\mathfrak{U})^{\prime}=\{\lambda I\}$.

\section{Strongly Cyclic *-Representations and States of *-Algebras}

Just as in the Gelfand-Segal construction for $C^{*}$-algebras, there is for each state $\omega$ of a ${ }^{*}$-algebra $\mathfrak{A}$ a strongly cyclic *-representation $\pi$ of $\mathfrak{A}$ on a Hilbert space $\mathfrak{H}$ with a cyclic vector $f_{0} \in \mathfrak{H}$ such that $\omega(A)=\left(f_{0}, \pi(A) f_{0}\right)$ for all $A \in \mathfrak{A}$. The representation $\pi$ is determined by $\omega$ up to unitary equivalence. The proof of this statement follows from the proof of the Wightman reconstruction theorem in quantum field theory $[15$, Theorem $3-7$, p. 117-126]. Furthermore, as in the theory of $C^{*}$-algebra, the induced representation $\pi$ is irreducible if and only if the state $\omega$ is pure (see [9] for proof for $C^{*}$-algebras).

Even strongly cyclic *-representations of commutative *-algebras may have unexpected properties. Hilbert proved the existence of a polynomial $P(x, y)$ in two real variables such that

(i) $P(x, y)$ is of sixth degree,

(ii) $P(x, y)>0$ for all $(x, y) \in R^{2}$,

(iii) $P$ is not a finite sum of squares of real polynomials.

Using Hilbert's construction, Gelfand and Vilenkin [4, Chap. II., Sec. 7.2, p. 232-236] argue that there exists a state $\omega$ on the free commutative algebra on two hermitian generators $A$ and $B$ such that $\omega(P(A, B))<0$ with $P$ a polynomial satisfying conditions (i) (ii) and (iii) above. Hence in the representation induced by $\omega, \pi(P) \geq 0$ while $P(x, y) \geqq 0$ for all $(x, y) \in R^{2}$. To avoid this type of pathology we define the notion of strongly positive states on commutative *-algebras, those states which are non-negative on non-negative real polynomials of hermitian elements. 
Definition 6.1. Suppose $\pi$ is a *-representation of a $*$-algebra $\mathfrak{A}$ on a Hilbert space $\mathfrak{H}$. A vector $f \in \mathfrak{D}(\pi)$ is said to be cyclic if $\{\pi(\mathfrak{Q}) f\}$ is dense in $\mathfrak{H}$ and $f$ is said to be strongly cyclic if $\{\pi(\mathfrak{Q}) f\}$ is dense in $\mathfrak{D}(\pi)$ in the induced topology on $\mathfrak{D}(\pi)$. A *-representation $\pi$ is said to be cyclic if it has a cyclic vector and strongly cyclic if it has a strongly cyclic vector.

Suppose $\pi$ is a ${ }^{*}$-representation of a *algebra $\mathfrak{A}$ on a Hilbert space $\mathfrak{H}$ and $f \in \mathfrak{D}(\pi)$. Let $\pi_{1}=\pi \mid\{\pi(\mathfrak{U}) f\}$. Then it is easily seen that $f$ is strongly cyclic if and only if the closure of $\pi_{1}$ is an extension of $\pi$ (i.e., $\pi_{1} \supset \pi$ ).

Definition 6.2. A state of a *-algebra $\mathfrak{A}$ with unit $I$ is a functional on satisfying the requirements.

(i) $\omega(\alpha A+B)=\alpha \omega(A)+\omega(B)$,

(ii) $\omega\left(A^{*} A\right) \geq 0$,

(iii) $\omega(I)=1$

for all $A, \mathrm{~B} \in \mathfrak{A}$ and complex numbers $\alpha$. A state $\omega$ is pure if it can not be written in the form $\omega=\lambda \omega_{1}+(1-\lambda) \omega_{2}$ with $\omega_{1} \neq \omega_{2}$ states of $\mathfrak{U}$ and $0<\lambda<1$.

Theorem 6.3. For each state $\omega$ of $a^{*}$-algebra $\mathfrak{A}$ with unit I there is a closed strongly cyclic *-representation $\pi$ of $\mathfrak{A}$ on a Hilbert space $\mathfrak{H}$ with strongly cyclic vector $f_{0} \in \mathfrak{D}(\pi)$ such that $\omega(A)=\left(f_{0}, \pi(A) f_{0}\right)$ for all $A \in \mathfrak{A}$. The representation $\pi$ is determined by $\omega$ up to unitary equivalence. Furthermore, the induced representation $\pi$ is irreducible if and only if $\omega$ is pure.

Proof. Since the proof of this theorem virtually a word for word reproduction of the corresponding theorem for $C^{*}$-algebras we give only an outline of the proof and refer to [6], [9] and [15, Theorem 3-7, p. 117]).

Let $\mathscr{I}=\left\{A \in \mathfrak{A} ; \omega\left(A^{*} A\right)=0\right\}$. One shows $\mathscr{I}$ is a left ideal and $\mathscr{I}^{*}=\left\{A \in \mathfrak{A} ; A^{*} \in \mathscr{I}\right\}$ is a right ideal. Let $Q=\mathfrak{U} / \mathscr{I}$ be the complex linear space of all equivalence classes $[A]=\{B \in \mathfrak{A} ; A-B \in \mathscr{I})$ of $\mathfrak{U}$ modulo $\mathscr{I}$. One shows $([A],[B])=\omega\left(A^{*} B\right)$ is a well defined inner product on $Q$. Let $\mathfrak{H}$ be the Hilbert space obtain upon completion of $Q$ relative to its inner product. We consider $Q$ as a dense subset of $\mathfrak{H}$.

We define a representation $\pi_{1}$ of $\mathfrak{A}$ on $Q$ by the relation $\pi_{1}(A)[B]$ $=[A B]$ for all $A, B \in \mathfrak{A}$. One shows $\pi_{1}$ is a well defined *-representation of $\mathfrak{A}$ on $\mathfrak{H}$ with domain $\mathfrak{D}\left(\pi_{1}\right)=Q$. Let $\pi$ be the closure of $\pi_{1}$ with domain $\mathfrak{D}(\pi)$. Let $f_{0}=[I] \in Q \subset \mathfrak{D}(\pi)$. We have $f_{0}$ is strongly cyclic since $\left\{\pi(\mathfrak{A}) f_{0}\right\}=Q$ is strongly dense in $\mathfrak{D}(\pi)$ and $\omega(A)=\left(f_{0} \pi(A) f_{0}\right)$ for all $A \in \mathfrak{A}$.

If $\pi$ and $\pi^{\prime}$ are strongly cyclic closed *-representations of $\mathfrak{A}$ on Hilbert spaces $\mathfrak{H}$ and $\mathfrak{H}^{\prime}$ with strongly cyclic vectors $f_{0}$ and $f_{0}^{\prime}$ such that $\left(f_{0}, \pi(A) f_{0}\right)$ $=\left(f_{0}^{\prime}, \pi^{\prime}(A) f_{0}^{\prime}\right)$ for all $A \in \mathfrak{A}$ then $\pi$ and $\pi^{\prime}$ are unitarily equivalent. To see this, one defines an isometry $U$ of $\left\{\pi(\mathfrak{A}) f_{0}\right\}$ onto $\left\{\pi^{\prime}(\mathfrak{A}) f_{0}^{\prime}\right\}$ by the relation $U \pi(A) f_{0}=\pi^{\prime}(A) f_{0}^{\prime}$ for all $A \in \mathfrak{A}$. One checks that $U$ is well defined, linear and isometric. Hence, $U$ can be extended to an isometry of $\mathfrak{H}$ onto 
$\mathfrak{H}^{\prime}$. The extension is also denoted by $U$. One checks that for a net $\left\{f_{\alpha} \in \pi(\mathfrak{A}) f_{0} ; \alpha \in I_{0}\right\} \lim _{\alpha} f_{\alpha}=f \in \mathfrak{D}(\pi)$ in the induced topology on $\mathfrak{D}(\pi)$ if and only if $\lim U f_{\alpha}=U f \in \mathfrak{D}\left(\pi^{\prime}\right)$ in the induced topology on $\mathfrak{D}\left(\pi^{\prime}\right)$. Since $f_{0}$ and $f_{0}^{\prime}$ are strongly cyclic $\left\{\pi(\mathfrak{Q}) f_{0}\right\}$ is dense in $\mathfrak{D}(\pi)$ and $\left\{\pi^{\prime}(\mathfrak{A}) f_{0}^{\prime}\right\}$ is dense in $\mathfrak{D}\left(\pi^{\prime}\right)$ in the induced topologies. It follows from these remarks and the fact that $\pi$ and $\pi^{\prime}$ are both closed that $U \mathfrak{D}(\pi)=\mathfrak{D}\left(\pi^{\prime}\right)$. Furthermore, we have $U \pi(A) f=\pi^{\prime}(A) U f$ for all $f \in \mathfrak{D}(\pi)$ and $A \in \mathfrak{A}$. Therefore, $\pi$ and $\pi^{\prime}$ are unitarily equivalent. Hence, the representation induced by a state $\omega$ is determined up to unitary equivalence.

Finally, we show that the induced representation $\pi$ is irreducible if and only if $\omega$ is pure. Suppose $\pi$ is a closed strongly cyclic representation of with strongly cyclic vector $f_{0} \in \mathfrak{D}(\pi)$ such that $\omega(A)=\left(f_{0}, \pi(A) f_{0}\right)$ for all $A \in \mathfrak{A}$.

First suppose $\omega$ is not pure. Then $\omega=\lambda \omega_{1}+(1-\lambda) \omega_{2}$ with $\omega_{1} \neq \omega_{2}$ states of $\mathfrak{A}$ and $0<\lambda<1$. Consider the bilinear form on $\left\{\pi(\mathfrak{Q}) f_{0}\right\}$ $x\left\{\pi(\mathfrak{U}) f_{0}\right\}$ defined by

$$
\left\langle\pi(A) f_{0}, \pi(B) f_{0}\right\rangle=\lambda \omega_{1}\left(A^{*} B\right)
$$

Using the fact that $\omega\left(A^{*} A\right)>\lambda \omega_{1}\left(A^{*} A\right)>0$ for all $A \in \mathfrak{A}$ one shows that the above bilinear form uniquely defines a positive operator $0<C<\lambda I$ such that

$$
\left\langle\left(\pi(A) f_{0}, \pi(B)\right) f_{0}\right\rangle=\left(\pi(A) f_{0}, C \pi(B) f_{0}\right)
$$

and

$$
\left(\pi\left(B_{1}\right) f_{0}, C \pi(A) \pi\left(B_{2}\right) f_{0}\right)=\left(\pi\left(A^{*}\right) \pi\left(B_{1}\right) f_{0}, C \pi\left(B_{2}\right) f_{0}\right)
$$

for all $A, B, B_{1}, B_{2} \in \mathfrak{A}$. Since $\pi(A)$ and $\pi\left(A^{*}\right)$ are continuous in the induced topology and $\left\{\pi(\mathfrak{Q}) f_{0}\right\}$ is dense in $\mathfrak{D}(\pi)$ in the induced topology, the above relation may be extended to all of $\mathfrak{D}(\pi)$. Hence, we have $(f, C \pi(A) g)=\left(\pi\left(A^{*}\right) f, C g\right)$ for all $f, g \in \mathfrak{D}(\pi)$ and $A \in \mathfrak{U}$. Hence $C \in \pi(\mathfrak{l})^{\prime}$ and $C \neq \lambda I$ since $\omega_{1} \neq \omega$.

Conversely, if $\pi$ is not irreducible there is an operator $C_{1} \in \pi(\mathfrak{l})^{\prime}$ with $C_{1} \notin\{\lambda I ; \lambda \in C\}$. Then for $\alpha=1$ or $\alpha=i, C_{2}=\left(\alpha C_{1}+\bar{\alpha} C_{1}^{*}\right) \notin\{\lambda I ; \lambda \in C\}$ and $C_{2} \in \pi(\mathfrak{Q})^{\prime}$. Let $C=\left(2\left\|C_{2}\right\|\right)^{-1}\left(\left\|C_{2}\right\| I+C_{2}\right)$. We have $0 \leqq C \leqq I$, $C \notin\{\lambda I ; \lambda \in C\}, C \in \pi(\mathfrak{A l})^{\prime}$. Note $\left(f_{0}, C f_{0}\right)>0$ since $\left(f_{0}, C f_{0}\right)=0$ implies $\left(\pi(A) f_{0}, C \pi(A) f_{0}\right)=\left(\pi\left(A^{*} A\right) f_{0}, C f_{0}\right)=0$ for all $A \in \mathfrak{A}$ which implies $C=0$. We have

$$
\begin{aligned}
\left(f_{0}, \pi\left(A^{*} A\right) C f_{0}\right) & =\left(\pi(A) f_{0}, C \pi(A) f_{0}\right)>0, \\
\left.\left(f_{0}, \pi\left(A^{*} A\right)\right)(I-C) f_{0}\right) & =\left(\pi(A) f_{0},(I-C) \pi(A) f_{0}\right)>0
\end{aligned}
$$

for all $A \in \mathfrak{A}$. Hence, $\omega_{1}(A)=\left(f_{0}, C f_{0}\right)^{-1}\left(f_{0}, \pi(A) C f_{0}\right)$ and $\omega_{2}(A)$ $=\left(f_{0},(I-C) f_{0}\right)^{-1}\left(f_{0}, \pi(A)(I-C) f_{0}\right)$ for all $A \in \mathfrak{A}$ are states of $\mathfrak{A}$ and 
$\omega=\lambda \omega_{1}+(1-\lambda) \omega_{2}$ with $\lambda=\left(f_{0}, C f_{0}\right)$. Since $C \notin\{\alpha I ; \alpha \in C\}$ we have $\omega_{1} \neq \omega_{2}$. Hence $\omega$ is not pure. This completes the outline of the proof of the theorem.

In the next section we will study strongly cyclic self-adjoint representations of commutative *-algebras. As pointed out in the beginning of this section Gelfand and Vilenkin have shown the existence of a state $\omega$ of the free commutative algebra on two hermitian generators $A$ and $B$ and a sixth degree polynomial $P(A, B) \in \mathfrak{A}$ such that $P(x, y)>0$ for all $(x, y) \in R^{2}$ and $\omega(P(A, B))<0$. In order to distinguish against such states we introduce the following definition.

Definition 6.4. Suppose $\omega$ is a state of a commutative *-algebra $\mathfrak{A}$. We say $\omega$ is strongly positive if for every finite set of hermitian elements $A_{1}, A_{2}, \ldots, A_{n}$ and every real positive polynomial $P\left(x_{1}, \ldots, x_{n}\right) \geqq 0$ for all $\left(x_{1}, \ldots, x_{n}\right) \in R^{n}$ we have $\omega\left(P\left(A_{1}, \ldots, A_{n}\right)\right) \geqq 0$. A *-representation of $\mathfrak{A}$ is said to be strongly positive if each vector state of $\pi$ is strongly positive (equivalently, if $\pi\left(P\left(A_{1}, \ldots, A_{n}\right)\right) \geqq 0$ for all positive polynomials of hermitian elements).

We remark that the cyclic representation induced by a strongly positive state of a commutative *-algebra is strongly positive. In the next section we shall need the following lemma which gives a well known necessary and sufficient condition for the moment problem to have a solution.

Lemma 6.5. Suppose $\mathfrak{A}$ is the free commutative *-algebra on $n$-hermitian generators $A_{1}, \ldots, A_{n}$ and suppose $\omega$ is a state of $\mathfrak{A}$. Then, a necessary and sufficient condition that there exists a regular Borel measure $\mu$ (not necessarily unique) on $R^{n}$ such that

$$
\omega\left(P\left(A_{1}, \ldots, A_{n}\right)\right)=\int P\left(x_{1}, \ldots, x_{n}\right) d \mu\left(x_{1}, \ldots, x_{n}\right)
$$

for all $P \in \mathfrak{A}$ is that $\omega$ be strongly positive.

Proof. For the proof we refer to [12].

\section{Standard Representations of Commutative *-Algebras}

In this section we define standard representations of commutative *-algebras. Intuitively, we mean by a standard representation of a commutative algebra a self-adjoint representation of the algebra by an algebra of functions on a measure space. Example 1 of Section $V$ is an example of a standard representation of a commutative *-algebra. To construct faithful standard representations of commutative *-algebras with an uncountable number of generators one has to deal with rather large measure spaces. We avoid such measure theoretic problems by 
defining standard representations in terms which can be stated as easily for finitely generated algebras as for infinitely generated algebras. We show that for a self-adjoint representation $\pi$ of a commutative *-algebra $\mathfrak{U}$, the following statements are equivalent:

(i) $\pi(A)^{*}=\overline{\pi(A)}$ for all hermitian $A \in \mathfrak{A}$,

(ii) $\pi(A)^{*}=\overline{\pi\left(A^{*}\right)}$ for all $A \in \mathfrak{A}$,

(iii) $\pi(\mathfrak{Q})^{\prime \prime}$ is commutative

where $\pi(\mathfrak{R})^{\prime \prime}$ is the commutant of $\pi(\mathfrak{Q})^{\prime}$. We define a standard representation of $\mathfrak{A}$ as a self-adjoint representation satisfying any one and, therefore, all of these statements. The reader may verify that a standard representation of a finitely generated commutative *-algebra is unitarily equivalent to a self-adjoint representation by an algebra of functions on a measure space.

We show in Theorem 7.3 that self-adjoint representations induced by strongly positive states are standard. Finally, we show that if $\mathfrak{A}$ is a commutative *-algebra which is dominated by a countably generated *-subalgebra. Then every standard representation of $\mathfrak{A}$ is a direct sum of strongly cyclic self-adjoint representations of $\mathfrak{A}$. The proof is based on Nelson's theory of analytic vectors [7] and techniques developed by Reed in [8].

Theorem 7.1. Suppose $\pi$ is a self-adjoint representation of a commutative *-algebra $\mathfrak{A}$. Then, the following statements are equivalent.

(i) $\pi(A)^{*}=\overline{\pi(A)}$ for all hermitian $A \in \mathfrak{U}$,

(ii) $\pi(A)^{*}=\overline{\pi\left(A^{*}\right)}$ for all $A \in \mathfrak{A}$,

(iii) $\pi(\mathfrak{A})^{\prime \prime}$ is commutative.

Proof. We prove (i) $\Rightarrow$ (ii) $\Rightarrow$ (iii) $\Rightarrow$ (i).

Suppose $\pi$ is a self-adjoint representation of a commutative *-algebra $\mathfrak{U}$ and statement (i) is true. To prove statement (ii) is valid it suffices to show $\mathfrak{D}\left(\overline{\left.\pi\left(A^{*}\right)\right)}=\mathfrak{D}\left(\pi(A)^{*}\right)\right.$ for all $A \in \mathfrak{A}$ since $\pi(A)^{*} \supset \overline{\pi\left(A^{*}\right)}$ for all $A \in \mathfrak{A}$. It is well known from the theory of the polar decomposition of a closed operator (see e.g. [3, Chap. XII, Sec. 7, p. 1245-1250]) that if $C$ is a closed operator on a Hilbert space $\mathfrak{S}$ then $H=C^{*} C$ is self-adjoint and $\mathfrak{D}\left(H^{\frac{1}{2}}\right)$ $=\mathfrak{D}(C)$ where $H^{\frac{1}{2}}$ is the positive square root of $H$. Suppose $A \in \mathfrak{A}$ and let $H_{1}=\pi\left(A^{*}\right)^{*} \overline{\pi\left(A^{*}\right)}$ and $H_{2}=\overline{\pi(A)} \pi(A)^{*}$. It follows that $\mathfrak{D}\left(\overline{\left.\pi\left(A^{*}\right)\right)}\right.$ $=\mathfrak{D}\left(H_{1}^{\frac{1}{2}}\right)$ and $\mathfrak{D}\left(\pi(A)^{*}\right)=\mathfrak{D}\left(H_{2}^{\frac{1}{2}}\right)$. We show $H_{1}=H_{2}$. Clearly, we have $H_{1}=\pi\left(A^{*}\right)^{*} \overline{\pi\left(A^{*}\right)} \supset \overline{\pi\left(A A^{*}\right)}$ and $H_{2}=\overline{\pi(A)} \pi(A)^{*} \supset \overline{\pi\left(A A^{*}\right)}$. Since $A A^{*}$ is hermitian we have $\overline{\pi\left(A A^{*}\right)}$ is self-adjoint. Since self-adjoint operators are maximal (i.e., they have no proper hermitian extensions) it follows that $H_{1}=\overline{\pi\left(A A^{*}\right)}=H_{2}$. Hence, $\mathfrak{D}\left(\pi(A)^{*}\right)=\mathfrak{D}\left(H_{2}^{\frac{1}{2}}\right)=\mathfrak{D}\left(H_{1}^{\frac{1}{2}}\right)=\mathfrak{D} \overline{\left(\pi\left(A^{*}\right)\right)}$ and hence $\pi(A)^{*}=\overline{\pi\left(A^{*}\right)}$ for all $A \in \mathfrak{U}$. Hence, we have (i) $\Rightarrow$ (ii). 
Next suppose statement (ii) is true. Then, if $A=A^{*} \in \mathfrak{A}, \overline{\pi(A)}$ is selfadjoint. Let $\left\{E_{A}(\lambda) ;-\infty<\lambda<+\infty\right\}$ be the spectral resolution of $\overline{\pi(A)}$ and let $R=\left\{E_{A}(\lambda) \text {; all } A=A^{*} \in \mathfrak{A},-\infty<\lambda<+\infty\right\}^{\prime \prime}$ be the von Neumann algebra generated by all the spectral projections of all the selfadjoint $\overline{\pi(A)}, A=A^{*} \in \mathfrak{A}$. We claim $R=\pi(\mathfrak{H})^{\prime \prime}$. Clearly, we have $C \in \pi(\mathfrak{H})^{\prime}$ if and only if $C$ commutes with each hermitian $\pi(A) \in \pi(\mathfrak{Q})$. Since these operators are essentially self-adjoint we have by the remark of Section I that $C \in \pi(\mathfrak{Q})^{\prime}$ if and only if $C E_{A}(\lambda)=E_{A}(\lambda) C$ for all $A=A^{*} \in \mathfrak{A}$ and $-\infty<\lambda<+\infty$. Hence, we have $\pi(\mathfrak{A})^{\prime}=R^{\prime}$ and $\pi(\mathfrak{A})^{\prime \prime}=R^{\prime \prime}=R$.

We show $\pi(\mathfrak{Q})^{\prime \prime}=R$ is commutative by showing the projections which generate $R$ are commutative. Suppose $A, B \in \mathfrak{A}$ are hermitian and let $C=A+i B$. Let $H_{1}=\pi(C)^{*} \overline{\pi(C)}$ and $H_{2}=\overline{\pi(C)} \pi(C)^{*}$. We have $H_{1}$ and $H_{2}$ are self-adjoint and $H_{1}=\pi(C)^{*} \overline{\pi(C)} \supset \overline{\pi\left(C^{*} C\right)}$ and $H_{2}=\overline{\pi(C)} \pi(C)^{*}$ $\supset \overline{\pi\left(C C^{*}\right)}=\overline{\pi\left(C^{*} C\right)}$. Since $C^{*} C$ is hermitian we have $\overline{\pi\left(C^{*} C\right)}$ is selfadjoint from statement (ii). By the maximality of self-adjoint operators we have $H_{1}=\overline{\pi\left(C^{*} C\right)}=H_{2}$. Hence, $\pi(C)^{*} \overline{\pi(C)}=\overline{\pi(C)} \pi(C)^{*}$ and $\overline{\pi(C)}$ is normal. Let

$$
\begin{aligned}
& A_{1}=\frac{1}{2}\left(\overline{\left.\overline{\pi(C)}+\pi(C)^{*}\right)},\right. \\
& A_{2}=\frac{1}{2 i}\left(\overline{\overline{\pi(C)}-\pi(C)^{*}}\right) .
\end{aligned}
$$

By the remark of Section I we have $A_{1}$ and $A_{2}$ are self-adjoint operators with mutually commuting spectral projections. Clearly, we have $A_{1} \supset \overline{\pi(A)}$ and $A_{2} \supset \overline{\pi(B)}$. By the maximality of self-adjoint operators we have $A_{1}=\overline{\pi(A)}$ and $A_{2}=\overline{\pi(B)}$. Hence, it follows that $E_{A}(\lambda) E_{B}(\mu)=E_{B}(\mu) E_{A}(\lambda)$ for all hermitian $A, B \in \mathfrak{A}$ and $-\infty<\lambda, \mu<+\infty$. Hence, $R=\pi(\mathfrak{l})^{\prime \prime}$ is commutative and (ii) $\Rightarrow$ (iii).

Finally, suppose statement (iii) is true. Suppose $A \in \mathfrak{A}$ is hermitian and let $U H=\overline{(\pi(A+i I))}$ be the unique polar decomposition of $\overline{\pi(A+i I)}$ where $H \geqq 0$ is self-adjoint and $U$ is a partial isometry from

$$
\overline{\operatorname{Range} \pi(A+i I)})^{*} \text { to } \overline{\operatorname{Range} \pi(A+i I)} \text {. }
$$

We claim $U \in \pi(\mathfrak{A})^{\prime \prime}$. Suppose $V \in \pi(\mathfrak{A})^{\prime}$ is unitary. Since $\pi$ is selfadjoint we have $V \mathfrak{D}(\pi) \subset \mathfrak{D}(\pi)$ and $V^{*} \pi(A+i I) V f=\pi(A+i I) f$ for all $f \in \mathfrak{D}(\pi)$. Hence $V^{*} \overline{\pi(A+i I)} V=\overline{\pi(A+i I)}$ and $V^{*} \overline{\pi(A+i I)} V=\left(V^{*} U V\right)$ $\cdot\left(V^{*} H V\right)=U H$. By the uniqueness of the polar decomposition we have $V^{*} U V=U$ and $V^{*} H V=H$. Since $\pi$ is self-adjoint $\pi(\mathfrak{2})^{\prime}$ is a von Neumann algebra. Hence, $\pi(\mathfrak{L})^{\prime}$ is generated by its unitary elements and, therefore, $U \in\left(\pi(\mathfrak{H})^{\prime}\right)^{\prime}=\pi(\mathfrak{l})^{\prime \prime}$.

Since $\pi(\mathfrak{A})^{\prime \prime}$ is commutative we have $U U^{*}=U^{*} U$ and, hence,

$$
\overline{\text { Range } \pi(A+i I)}=\overline{\text { Range } \pi(A+i I)^{*}} \text {. }
$$


We have $f$ orthogonal to $\overline{\text { Range } \pi(A+i I})$ if and only if $f \in \mathfrak{D}\left(\pi(A)^{*}\right)$ and $\pi(A)^{*} f=$ if and we have $g$ orthogonal to Range $\pi(A+i I)^{*}$ if and only if $g \in \mathfrak{D}(\overline{\pi(A)})$ and $\overline{\pi(A)} g=-i g$. Since these ranges are equal we have $f \in \mathfrak{D}\left(\pi(A)^{*}\right)$ and $\pi(A)^{*} f=i f$ if and only if $f \in \mathfrak{D} \overline{(\pi(A))}$ and $\overline{\pi(A)} f$ $=\pi(A)^{*} f=-i f$. Hence, $\overline{\text { Range } \pi(A+i I)}=\mathfrak{H}$. Repeating this argument for $\pi(A-i I)$ one finds $\overline{\text { Range } \pi(A-i I)}=\mathfrak{H}$. Hence, the deficiency spaces $\mathfrak{D}_{+}$and $\mathfrak{D}_{-}$for $\overline{\pi(A)}$ are $\mathfrak{D}_{+}=\mathfrak{D}_{-}=\{0\}$. Hence, $\overline{\pi(A)}$ is self-adjoint. Since $A$ is an arbitrary hermitian element we have (iii) $\Rightarrow$ (i). This completes the proof of the theorem.

Definition 7.2. Suppose $\pi$ is a $*$-representation of a commutative *-algebra $\mathfrak{A}$. $\pi$ is said to be standard if $\pi$ is a self-adjoint representation satisfying conditions (i), (ii) and (iii) of Theorem 7.1.

Theorem 7.3. Suppose $\pi$ is a strongly cyclic self-adjoint *-representation of a commutative *algebra $\mathfrak{A}$ on a Hilbert space $\mathfrak{H}$. Suppose $f_{0}$ is a strongly cyclic vector with $\left\|f_{0}\right\|=1$ and $\omega(A)=\left(f_{0}, \pi(A) f_{0}\right)$ for all $A \in \mathfrak{U}$ is the state determined by $f_{0}$. Then $\pi$ is standard if and only if $\omega$ is strongly positive.

Proof. First suppose $\pi$ is standard and $\left\{A_{i} \in \mathfrak{A} ; i=1, \ldots, n\right\}$ are hermitian elements of $\mathfrak{H}$. Since $\pi$ is standard $\overline{\pi\left(A_{i}\right)}$ is self-adjoint and the spectral projections $E_{i}(\lambda)$ of $\overline{\pi\left(A_{i}\right)}$ commute with the spectral projections $E_{j}(\lambda)$ of $\overline{\pi\left(A_{j}\right)}$ for $i, j=1, \ldots, n$. Let $E\left(\lambda_{1}, \ldots, \lambda_{n}\right)=E_{1}\left(\lambda_{1}\right) E_{2}\left(\lambda_{2}\right), \ldots, E_{n}\left(\lambda_{n}\right)$ be the joint spectral resolution for $\overline{\pi\left(A_{1}\right)}, \ldots, \overline{\pi\left(A_{n}\right)}$. The functional calculus for self-adjoint operators then shows that for $f_{0} \in \mathfrak{D}(\pi)$ and $P=P\left(A_{1}, \ldots, A_{n}\right)$ a polynomial in $\left(A_{1}, \ldots, A_{n}\right)$ we have

$$
\left(f_{0}, \pi(P) f_{0}\right)=\int P\left(\lambda_{1}, \ldots, \lambda_{n}\right) d\left(f_{0}, E\left(\lambda_{1}, \ldots, \lambda_{n}\right) f_{0}\right) .
$$

Hence, if $P\left(\lambda_{1}, \ldots, \lambda_{n}\right) \geqq 0$ for all real $\lambda_{1}, \ldots, \lambda_{n}$ we have $\omega(P)=\left(f_{0}, \pi(P) f_{0}\right)$ $\geqq 0$. Hence, $\omega$ is strongly positive.

Conversely, suppose $\omega$ is strongly positive. We show $\pi\left(A_{0}\right)$ is self-adjoint for all hermitian $A_{0} \in \mathfrak{A}$. Suppose $A_{0}=A_{0}^{*} \in \mathfrak{A}$. Let $S=\left\{A_{1}, \ldots, A_{n}\right\}$ be a finite set of elements of $\mathfrak{A}$ and let $\mathfrak{B}_{s}$ the commutative *-subalgebra of $\mathfrak{U}$ consisting of all polynomials in $A_{0}$ and $A_{i} \in S$; $i=1, \ldots, n$.

Let $\mathfrak{M}_{s}=\left\{\overline{\pi\left(\mathfrak{B}_{s}\right) f_{0}}\right\}$ and let $\pi_{s}^{\prime}$ be the restriction of $\pi$ to $\mathfrak{B}_{s}$ and $\left\{\pi\left(\mathfrak{A}_{s}\right) f_{0}\right\}$, i.e., $\pi_{s}^{\prime}(A) f=\pi(A) f$ for all $A \in \mathfrak{B}_{s}$ and $f \in\left\{\pi\left(\mathfrak{B}_{s}\right) f_{0}\right\}$. Let $\pi_{s}$ be the closure of $\pi_{s}^{\prime}$. Clearly $\pi_{s}$ is a closed strongly cyclic *-representation of $\mathfrak{B}_{s}$ induced by the state $\omega_{s}=\omega \mid \mathfrak{B}_{s}$. In fact, we have $\omega_{s}(A)=\left(f_{0}, \pi_{s}(A) f_{0}\right)$ for all $A \in \mathfrak{B}_{s}$.

Since $\omega$ is strongly positive $\omega_{s}=\omega \mid \mathfrak{B}_{s}$ is strongly positive. Hence, by Lemma 6.5 there is a regular Borel measure $\mu$ (possibly not unique) on 
$R^{n+1}$ such that

$$
\omega_{s}\left(P\left(A_{0}, A_{1}, \ldots, A_{n}\right)\right)=\int P\left(x_{0}, x_{1}, \ldots, x_{n}\right) d \mu\left(x_{0}, x_{1}, \ldots, x_{n}\right)
$$

for all polynomials $P\left(A_{0}, A_{1}, \ldots, A_{n}\right) \in \mathfrak{B}_{s}$. Let $\mathfrak{H}_{s}=L^{2}\left(R^{n+1}, \mu\right)$ be the Hilbert space of all $\mu$-measurable square integrable functions on $R^{n+1}$ and let $\Phi_{s}$ be the standard representation of $\mathfrak{B}_{s}$ on $\mathfrak{H}_{s}$ defined the relations

$$
\begin{array}{r}
\left(\Phi_{s}\left(P\left(A_{0}, A_{1}, \ldots, A_{n}\right)\right) F\right)\left(x_{0}, x_{1}, \ldots, x_{n}\right) \\
\quad=P\left(x_{0}, x_{1}, \ldots, x_{n}\right) F\left(x_{0}, x_{1}, \ldots, x_{n}\right)
\end{array}
$$

for all $F \in \mathfrak{D}\left(\Phi_{s}\right)=\left\{F \in \mathfrak{H}_{s} ; P\left(x_{0}, x_{1}, \ldots, x_{n}\right) F\left(x_{0}, x_{1}, \ldots, x_{n}\right) \in \mathfrak{H}_{s}\right.$ for all polynomials $P\}$. As shown in Example 1 of Section $\mathrm{V}, \overline{\Phi_{s}\left(P^{*}\right)}=\Phi_{s}(P)^{*}$ for all $P \in \mathfrak{B}_{s}$. Hence, $\Phi_{s}$ is standard. Let $e_{0} \in \mathfrak{H}_{s}$ be the function $e_{0}\left(x_{0}, x_{1}, \ldots, x_{n}\right)=1$ and let $\mathfrak{N}_{s}=\left\{\overline{\Phi_{s}\left(\mathfrak{B}_{s}\right) e_{0}}\right\}$ and let $E_{s}$ be the hermitian projection onto $\mathfrak{N}_{s}$. Let $\tau_{s}$ be the closure of the restriction of $\Phi_{s}$ to $\left\{\Phi_{s}\left(\mathfrak{B}_{s}\right) e_{0}\right\}$. Note $\omega_{s}(A)=\left(e_{0}, \tau_{s}(A) e_{0}\right)$ for all $A \in \mathfrak{B}_{s}$. Since $e_{0}$ is strongly cyclic for $\tau_{s}, \tau_{s}$ is a closed $*$-representation induced by $\omega_{s}$.

Since $\pi_{s}$ and $\tau_{s}$ are closed *-representations induced by $\omega_{s}$ there is an isometry $U$ of $\mathfrak{M}_{s}$ onto $\mathfrak{N}_{s}$ such that $U \mathfrak{D}\left(\pi_{s}\right)=\mathfrak{D}\left(\tau_{s}\right)$ and $U^{*} \tau_{s}(A) U f$ $=\pi_{s}(A) f$ for all $A \in \mathfrak{B}_{s}$ and $f \in \mathfrak{D}\left(\pi_{s}\right)$ and, finally, $U f_{0}=e_{0}$ (see Theorem 6.3).

Since $\Phi_{s}$ is standard and $A_{0}=A_{0}^{*}$, we have $\overline{\Phi_{s}\left(A_{0}\right)}$ is self-adjoint and $\overline{\Phi_{s}\left(A_{0}+i I\right)}{ }^{-1}$ exists. Let $\left.C_{s}=U^{*} E_{s} \overline{\Phi_{s}\left(A_{0}+i I\right.}\right)^{-1} E_{s} U . C_{s}$ is defined on $\mathfrak{M}_{s} \subset \mathfrak{H}$. We extend $C_{s}$ to all of $\mathfrak{H}$ by defining $C_{s} f=0$ for $f \in \mathfrak{M}_{\mathrm{s}}^{\perp}$. A straight forward computation shows that

$$
\begin{gathered}
C_{s} \pi\left(A_{0}+i I\right) f_{0}=f_{0}, \\
\left(\pi\left(A^{*}\right) \pi\left(B_{1}\right) f_{0}, C_{s} \pi\left(B_{2}\right) f_{0}\right)=\left(\pi\left(B_{1}\right) f_{0}, C_{s} \pi(A) \pi\left(B_{2}\right) f_{0}\right)
\end{gathered}
$$

for all $A, B_{1}, B_{2} \in \mathfrak{B}_{s}$.

Hence, for each finite set $S$ of hermitian elements of $\mathfrak{A}$ there exists an operator $C_{s}$ on $\mathfrak{H}$ such that $\left\|C_{s}\right\| \leqq 1$ and $C_{s}$ satisfies relations (a) and (b) above. Hence, by the axiom of choice there is a net $S \rightarrow C_{s}$ of such operators where the sets $S$ are ordered by inclusion. Since the unit ball of $\mathfrak{B}(\mathfrak{H})$ is compact in the weak operator topology $[1$, Chap. IV, $\S 2$, Thm. 1, Cor. 3] the net $\left\{C_{s}\right\}$ has at least one cluster point in the weak topology. Let $C$ be a cluster point of $\left\{C_{s}\right\}$. We claim $C \in \pi(\mathfrak{A})^{\prime}$ and $C \pi\left(A_{0}+i I\right) f_{0}=f_{0}$.

Since $C_{s} \pi\left(A_{0}+i I\right) f_{0}=f_{0}$ for all $S$ it follows that $C \pi\left(A_{0}+i I\right) f_{0}=f_{0}$. We show $C \in \pi(\mathfrak{U})^{\prime}$. Suppose $A, B_{1}, B_{2} \in \mathfrak{U}$ and $\varepsilon>0$. Since $C$ is a weak cluster point of the set $\left\{C_{s}\right\}$ there is a finite set $S$ such that $A, B_{1}, B_{2} \in \mathfrak{B}_{s}$ and

$$
\begin{aligned}
& \left|\left(\pi\left(A^{*}\right) \pi\left(B_{1}\right) f_{0},\left(C_{s}-C\right) \pi\left(B_{2}\right) f_{0}\right)\right|<\varepsilon / 2, \\
& \left|\left(\pi\left(B_{1}\right) f_{0},\left(C_{s}-C\right) \pi(A) \pi\left(B_{2}\right) f_{0}\right)\right|<\varepsilon / 2 .
\end{aligned}
$$


Combining these inequalities with Eq. (b) we obtain

$$
\left|\left(\pi\left(A^{*}\right) \pi\left(B_{1}\right) f_{0}, C \pi\left(B_{2}\right) f_{0}\right)-\left(\pi\left(B_{1}\right) f_{0}, C \pi(A) \pi\left(B_{2}\right) f_{0}\right)\right|<\varepsilon .
$$

Since $\varepsilon$ is arbitrary we have

$$
\left(\pi\left(A^{*}\right) \pi\left(B_{1}\right) f_{0}, C \pi\left(B_{2}\right) f_{0}\right)=\left(\pi\left(B_{1}\right) f_{0}, C \pi(A) \pi\left(B_{2}\right) f_{0}\right)
$$

for all $A, B_{1}, B_{2} \in \mathfrak{A}$. Since $f_{0}$ is strongly cyclic there are for each $f, g \in \mathfrak{D}(\pi)$ sequences $\left\{B_{1 n}\right\}$ and $\left\{B_{2 n}\right\}$ such that $\pi\left(B_{1 n}\right) f_{0} \rightarrow f, \pi\left(A^{*}\right) \pi\left(B_{1 n}\right) f_{0} \rightarrow \pi\left(A^{*}\right) f$, $\pi\left(B_{2 n}\right) f_{0} \rightarrow g$ and $\pi(A) \pi\left(B_{2 n}\right) f_{0} \rightarrow \pi(A) g$ as $n \rightarrow \infty$. Hence, we have

$$
\begin{aligned}
\left(\pi\left(A^{*}\right) f, C g\right) & =\lim _{n \rightarrow \infty}\left(\pi\left(A^{*}\right) \pi\left(B_{1 n}\right) f_{0}, C \pi\left(B_{2 n}\right) f_{0}\right) \\
& =\lim _{n \rightarrow \infty}\left(\pi\left(B_{1 n}\right) f_{0}, C \pi(A) \pi\left(B_{2 n}\right) f_{0}\right) \\
& =(f, C \pi(A) g) .
\end{aligned}
$$

Hence, $C \in \pi(\mathfrak{Q})^{\prime}$. Since $\pi$ is self-adjoint we have from Lemma 4.6 that $C \mathfrak{D}(\pi) \subset \mathfrak{D}(\pi)$ and

$$
C \pi\left(A_{0}+i I\right) f_{0}=\pi\left(A_{0}+i I\right) C f_{0}=f_{0} .
$$

Hence, $f_{0} \in$ Range $\pi\left(A_{0}+i I\right)$ and $\pi(A) f_{0}=\pi(A) \pi\left(A_{0}+i I\right) C f_{0}=\pi\left(A_{0}+i I\right)$ - $\pi(A) C f_{0} \in$ Range $\pi\left(A_{0}+i I\right)$ for all $A \in \mathfrak{A}$. Since $f_{0}$ is cyclic it follows that the range of $\pi\left(A_{0}+i I\right)$ is dense in $\mathfrak{H}$. Repeating this argument for $\pi\left(A_{0}-i I\right)$ we have that the Range $\pi\left(A_{0}-i I\right)$ is dense in $\mathfrak{H}$. Hence, $\pi\left(A_{0}\right)$ had deficiency spaces $\mathfrak{D}_{+}=\mathfrak{D}_{-}=\{0\}$ and $\overline{\pi\left(A_{0}\right)}$ is essentially self-adjoint. Since $\overline{\pi\left(A_{0}\right)}$ is self-adjoint for arbitrary hermitian $A_{0}$ we have that $\pi$ is standard. This completes the proof of the Theorem.

Next we will show that every standard representation of a commutative *-algebra $\mathfrak{A}$ which is dominated by a countably generated *-subalgebra $\mathfrak{A}_{1}$, is the direct sum of strongly cyclic standard representations. The main part of the proof is in the following lemma.

Lemma 7.4. Suppose $\mathfrak{A}$ is a commutative *-algebra which is dominated by a countably generated ${ }^{*}$-subalgebra $\mathfrak{H}_{1}$. Suppose $\pi$ is a standard representation of $\mathfrak{A}$ on a Hilbert space $\mathfrak{H}$. Then $\pi$ is strongly cyclic if and only if $\pi(\mathfrak{Q})^{\prime \prime}$ is cyclic.

Proof. Suppose $\mathfrak{A}, \mathfrak{U}_{1}$ and $\pi$ are as stated in the lemma.

First suppose $\pi$ is cyclic with cyclic vector $f_{0} \in \mathfrak{D}(\pi)$. Suppose $g \in \mathfrak{H}$ and $\varepsilon>0$. Since $f_{0}$ is cyclic there are hermitian elements $A, B \in \mathfrak{A}$ such that $\left\|\pi(A+i B) f_{0}-g\right\|<\varepsilon / 2$. Let

$$
A_{n}=\int_{-n}^{n} \lambda d E_{A}(\lambda), \quad B_{n}=\int_{-n}^{n} \lambda d E_{B}(\lambda),
$$


where $E_{A}(\lambda)$ and $E_{B}(\lambda)$ are the spectral resolutions of $\overline{\pi(A)}$ and $\overline{\pi(B)}$. Since $f_{0} \in \mathfrak{D}(\overline{\pi(A))} \cap \mathfrak{D}(\overline{\pi(B))}$ there is an integer $r$ such that

$$
\left\|\left(A_{r}-\pi(A)\right) f_{0}\right\|<\varepsilon / 4, \quad\left\|\left(B_{r}-\pi(B)\right) f_{0}\right\|<\varepsilon / 4 .
$$

By the argument of Theorem 7.1, $E_{A}(\lambda), E_{B}(\lambda) \in \pi(\mathfrak{Q})^{\prime \prime}$ for all real $\lambda$. Hence, $C=A_{r}+i B_{r} \in \pi(\mathfrak{Q})^{\prime \prime}$. Now, we have

$$
\begin{aligned}
\left\|C f_{0}-g\right\|= & \left\|A_{r} f_{0}+i B_{r} f_{0}-g\right\| \\
& \leqq\left\|A_{r} f_{0}-\pi(A) f_{0}\right\|+\left\|B_{r} f_{0}-\pi(B) f_{0}\right\| \\
& +\left\|\pi(A+i B) f_{0}-g\right\| \\
& <\varepsilon / 4+\varepsilon / 4+\varepsilon / 2=\varepsilon .
\end{aligned}
$$

Hence, $g \in \mathfrak{H}$ may be approximated arbitrarily well by vectors $C f_{0}$ with $C \in \pi(\mathfrak{Q})^{\prime \prime}$. Hence, $\pi(\mathfrak{Q})^{\prime \prime}$ is cyclic with a cyclic vector $f_{0}$.

Next suppose $\pi(\mathfrak{Q})^{\prime \prime}$ is cyclic with cyclic vector $f_{0}$ and $\left\|f_{0}\right\|=1$. Since $\mathfrak{U}_{1} \subset \mathfrak{A}$ is countably generated $\mathfrak{U}_{1}$ has a countable algebraic basis $\left\{A_{n} ; A_{n}=A_{n}^{*}, n=1,2, \ldots\right\}$ i.e. each $A \in \mathfrak{A}$ is a finite linear combination of $A_{n}$. In fact, the $A_{n}$ may be chosen to be monomials in the generators of $\mathfrak{H}_{1}$. Let

$$
T_{n}(s)=\exp \left(-s \overline{\pi\left(A_{n}^{2}\right)}\right)=\int e^{-s \lambda^{2}} d E_{A_{n}}(\lambda)
$$

for $s \geqq 0$ and where $E_{A_{n}}(\lambda)$ is the spectral resolution of $\overline{\pi\left(A_{n}\right)}$. Since $T_{n}(s)$ converges strongly to $I$ as $s \rightarrow 0$ from above, there are positive numbers $\left\{s_{n} ; n=1,2, \ldots\right\}$ such that

$$
\left\|T_{n}\left(s_{n}\right) f_{0}-f_{0}\right\| \leqq 2^{-(n+1)} .
$$

Let

$$
Q_{n}=T_{1}\left(s_{1}\right) T_{2}\left(s_{2}\right) \ldots T_{n}\left(s_{n}\right) .
$$

Since the $Q_{n}$ form a decrasing sequence of positive operators it follows (see e.q. [2, Appendix II, p. 331]) $Q_{n}$ converges strongly to an operator $Q$ is $n \rightarrow \infty$. Let $g_{0}=Q f_{0}$, Note $g_{0} \neq 0$ since

$$
\begin{aligned}
\left\|g_{0}\right\| & \geqq\left\|f_{0}\right\|-\left\|f_{0}-g_{0}\right\| \\
& \geqq 1-\sum_{n=0}^{\infty}\left\|Q_{n+1} f_{0}-Q_{n} f_{0}\right\| \\
& \geqq 1-\sum_{n=0}^{\infty}\left\|Q_{n}\left(T_{n+1}\left(s_{n+1}\right)-I\right) f_{0}\right\| \\
& \geqq 1-\sum_{n=0}^{\infty}\left\|T_{n+1}\left(s_{n+1}\right) f_{0}-f_{0}\right\| \\
& \geqq 1-\sum_{n=1}^{\infty} 2^{-(n+1)}=\frac{1}{2} .
\end{aligned}
$$

We will show $g_{0} \in \mathfrak{D}(\pi)$ and $g_{0}$ is strongly cyclic for $\pi$. 
Clearly, $g_{0} \in \mathfrak{D}\left(Q_{n}^{-1}\right)=\mathfrak{D}\left(\exp \overline{\left(\pi\left(\sum_{i=1}^{n}\right) s_{i} A_{i}^{2}\right)}\right)$ for all $n=1,2, \ldots$ In fact, $Q_{n}^{-1} g_{0}=\lim _{m \rightarrow \infty} T_{n+1}\left(s_{n+1}\right) \ldots T_{m}\left(s_{m}\right) f_{0}$. Hence, $g_{0} \in \mathfrak{D}\left(\overline{\pi\left(A_{n}\right)}\right)$ for all $n=1,2, \ldots$. Since the $A_{n}$ span $\mathfrak{A}_{1}$ it follows that $g_{0} \in \mathfrak{D}(\overline{(\pi(B))}$ for all $B \in \mathfrak{U}_{1}$. Since $\mathfrak{U}_{1}$ dominates $\mathfrak{U}$ for each $A \in \mathfrak{A}$ there is a $B \in \mathfrak{U}_{1}$, such that $\|\pi(B) f\| \geqq\|\pi(A) f\|$ for all $f \in \mathfrak{D}(\pi)$. It follows that $\mathfrak{D} \overline{(\pi(B))} \subset \mathfrak{D} \overline{(\pi(A))}$. Hence, $g_{0} \in \mathfrak{D}(\pi(A))$ for all $A \in \mathfrak{A}$ and since $\pi$ is closed $g_{0} \in \mathfrak{D}(\pi)$.

Let $\mathfrak{R}=\left\{\pi(\mathfrak{Q}) g_{0}\right\}$ and let $\pi_{1}$ be the closure of the restriction of $\pi$ to $\mathfrak{N}$, i.e. $\pi_{1}=\pi^{\mid} \mathfrak{N}$. We will show $\pi_{1}=\pi$. We first show $\pi_{1}$ is self-adjoint.

Suppose $A=A^{*} \in \mathfrak{U}_{1}$ and $B \in \mathfrak{A}$. We show $\pi_{1}(B) g_{0}$ is an analytic vector for $\pi_{1}(A)$. Since $\mathfrak{U}_{1}$ dominates $\mathfrak{A}$ there is a $B_{0} \in \mathfrak{A}_{1}$ such that $\left\|\pi_{1}\left(B_{0}\right) f\right\| \geqq\left\|\pi_{1}(B) f\right\|$ for all $f \in \mathfrak{D}\left(\pi_{1}\right)$. Since $A, B_{0} \in \mathfrak{A}_{1}$ we can express $A$ and $B_{0}$ in the form $A=\sum_{i=1}^{m} \alpha_{i} A_{i}$ and $B_{0}=\sum_{i=1}^{m} \beta_{i} A_{i}$. We have

$$
\begin{aligned}
& \sum_{n=0}^{\infty}\left\|\pi_{1}\left(A^{n}\right) \pi_{1}(B) g_{0}\right\| s^{n} / n ! \\
& \quad=\sum_{n=0}^{\infty}\left\|\pi_{1}(B) \pi_{1}\left(A^{n}\right) g_{0}\right\| s^{n} / n ! \\
& \quad \leqq \sum_{n=0}^{\infty}\left\|\pi_{1}\left(B_{0}\right) \pi_{1}\left(A^{n}\right) g_{0}\right\| s^{n} / n ! \\
& \quad=\sum_{n=0}^{\infty}\left\|\pi_{1}\left(B_{0} A^{n}\right) Q_{m} Q_{m}^{-1} g_{0}\right\| s^{n} / n ! \\
& \quad \leqq\left\|Q_{m}^{-1} g_{0}\right\| \sum_{n=0}^{\infty}\left\|\pi_{1}\left(B_{0} A^{n}\right) Q_{m}\right\| s^{n} / n ! .
\end{aligned}
$$

We estimate

$$
\begin{aligned}
\left\|\pi_{1}\left(B_{0} A^{n}\right) Q_{m}\right\| & \leqq \sup \left(\left|\sum_{i=1}^{m} \beta_{i} x_{i}\right|\left|\sum_{i=1}^{m} \alpha_{i} x_{i}\right|^{n} \exp \left(-\sum_{i=1}^{m} s_{i} x_{i}^{2}\right) \mid\right. \\
& \leqq\|\hat{\beta}\|\|\hat{\alpha}\|^{n} \sup _{x \in R}\left|x^{n+1} e^{-q x^{2}}\right| \\
& \leqq\|\hat{\beta}\|\|\hat{\alpha}\|^{n}\left(\frac{n+1}{2 q}\right)^{\frac{n+1}{2}}
\end{aligned}
$$

where $q=\min \left(s_{1}, \ldots, s_{m}\right)$ and $\|\hat{\alpha}\|=\left(\sum_{i=1}^{m}\left|\alpha_{i}\right|^{2}\right)^{\frac{1}{2}}$ and $\|\hat{\beta}\|=\left(\sum_{i=1}^{m}\left|\beta_{i}\right|^{2}\right)^{\frac{1}{2}}$. 
Hence, we have

$$
\begin{aligned}
& \sum_{n=0}^{\infty}\left\|\pi_{1}\left(A^{n}\right) \pi_{1}(B) g_{0}\right\| s^{n} / n ! \\
& \quad \leqq\left\|Q_{m}^{-1} g_{0}\right\|\|\hat{\beta}\| \sum_{n=0}^{\infty} \frac{s^{n}\|\hat{\alpha}\|^{n}}{n !}\left(\frac{n+1}{2 q}\right)^{\frac{n+1}{2}} \\
& \quad<+\infty \text { for all } s \geqq 0 .
\end{aligned}
$$

Hence, $\pi_{1}(B) g_{0}$ is an analytic vector for $\pi_{1}(A)$ for all $B \in \mathfrak{A}$. Since $g_{0}$ is cyclic for $\pi_{1}$ it follows that for each hermitian $A \in \mathfrak{A}_{1} \pi_{1}(A)$ has a dense set of analytic vectors. It follows from a theorem of Nelson [7, Lemma 5.1] that $\pi_{1}(A)$ is essentially self-adjoint for all hermitian $A \in \mathfrak{A}_{1}$.

Let $\pi_{2}(A)=\pi_{1}(A)$ for all $A \in \mathfrak{A}_{1}$ and let $\mathfrak{D}\left(\pi_{2}\right)=\mathfrak{D}\left(\pi_{1}\right)$. Since $\mathfrak{A}_{1}$ dominates $\mathfrak{A}$ for every $A \in \mathfrak{U}$ there is a $B \in \mathfrak{U}_{1}$ such that $\mathfrak{D} \overline{\left(\pi_{1}(B)\right)}$ $\subset \mathfrak{D} \overline{\left(\pi_{1}(A)\right)}$. Hence,

Hence

$$
\bigcap_{B \in \mathfrak{Q}_{1}} \mathfrak{D} \overline{\left(\pi_{2}(B)\right)} \subset \bigcap_{A \in \mathfrak{Q}} \mathfrak{D} \overline{\left(\pi_{1}(A)\right)}=\mathfrak{D}\left(\pi_{1}\right)=\mathfrak{D}\left(\pi_{2}\right) .
$$

$$
\mathfrak{D}\left(\pi_{2}\right)=\bigcap_{B \in \mathfrak{Q}_{1}} \mathfrak{D} \overline{\left(\pi_{2}(B)\right)}
$$

and $\pi_{2}$ is closed. We have shown that $\overline{\pi_{1}(A)}=\overline{\pi_{2}(A)}$ is self-adjoint for all hermitian $A \in \mathfrak{A}_{1}$. Hence, we have

$$
\begin{aligned}
\mathfrak{D}\left(\pi_{2}\right) & =\bigcap_{A \in \mathfrak{Q}_{1}} \mathfrak{D} \overline{\left(\pi_{2}(A)\right)}=\bigcap_{A \in \mathfrak{H}_{1}, A=A^{*}} \mathfrak{D} \overline{(\pi(A))} \\
& =\bigcap_{A \in \mathfrak{U}_{1}, A=A^{*}} \mathfrak{D}\left(\pi_{2}(A)^{*}\right)=\bigcap_{A \in \mathfrak{Q}_{1}} \mathfrak{D}\left(\pi(A)^{*}\right) \\
& =\mathfrak{D}\left(\pi_{2}^{*}\right) .
\end{aligned}
$$

Hence, $\pi_{2}$ is self-adjoint. It then follows immediately from Lemma 4.9 that $\pi_{1}$ is a self-adjoint representation of $\mathfrak{A}$ on $\overline{\mathfrak{R}}$. Hence, $\pi_{1}$ is a selfadjoint restriction of $\pi$ and by Theorem $4.7 \overline{\mathfrak{N}}=E \mathfrak{S}$ and $\mathfrak{D}\left(\pi_{1}\right)=E \mathfrak{D}(\pi)$ where $E \in \pi(\mathfrak{Q})^{\prime}$ is an hermitian projection.

We show $E=I$ and, hence, $\pi_{1}=\pi$. We first show $f_{0} \in \overline{\mathfrak{N}}$. Suppose $\varepsilon>0$. We have

$$
\begin{aligned}
\left\|Q_{n}^{-1} g_{0}-f_{0}\right\|= & \lim _{m \rightarrow \infty}\left\|T_{n+1}\left(s_{n+1}\right) \ldots T_{m}\left(s_{m}\right) f_{0}-f_{0}\right\| \\
& \leqq \sum_{m=n+1}^{\infty}\left\|\left(T_{m}\left(s_{m}\right)-I\right) f_{0}\right\| \\
& \leqq \sum_{m=n+1}^{\infty} 2^{-(m+1)}=2^{-(n+1)} .
\end{aligned}
$$


Hence, there is an integer $n$ such that $\left\|Q_{n}^{-1} g_{0}-f_{0}\right\| \leqq 2^{-(r+1)}<\varepsilon / 2$. Let $E(\lambda)$ be the spectral resolution of $Q_{n}^{-1}$ and let

$$
S_{\sigma}=\int_{0}^{\sigma} \lambda d E(\lambda)
$$

Since $g_{0} \in \mathfrak{D}\left(Q_{n}^{-1}\right)$ there a $\sigma$ such that $\left\|S_{\sigma} g_{0}-Q_{n}^{-1} g_{0}\right\|<\varepsilon / 2$. We have $S_{\sigma} \in \pi(\mathfrak{Q})^{\prime \prime}$ and

$$
\left\|S_{\sigma} g_{0}-f_{0}\right\| \leqq\left\|S_{\sigma} g_{0}-Q_{n}^{-1} g_{0}\right\|+\left\|Q_{n}^{-1} g_{0}-f_{0}\right\|<\varepsilon / 2+\varepsilon / 2=\varepsilon .
$$

Hence, $f_{0}$ can be approximated uniformly be vectors $S_{\sigma} g_{0}$ with $S_{\sigma} \in \pi(\mathfrak{Q})^{\prime \prime}$. Since $g_{0} \in \overline{\mathfrak{N}}=E \mathfrak{H}$ with $E \in \pi(\mathfrak{Q})^{\prime}$ it follows that $f_{0} \in \overline{\mathfrak{N}}$. Since $f_{0}$ is cyclic for $\pi(\mathfrak{A})^{\prime \prime}$ and $\overline{\mathfrak{N}}$ is invariant under $\pi(\mathfrak{R})^{\prime \prime}$, it follows that $\overline{\mathfrak{A}}=\mathfrak{H}$ and $E=I$. Hence, $\pi_{1}=\pi$ and $g_{0}$ is strongly cyclic for $\pi$. This completes the proof of the theorem.

We define the direct sum of representations of *algebra in the obvious manner. Suppose $\left\{\pi_{\alpha} ; \alpha \in I_{0}\right\}$ is a collection of closed ${ }^{*}$-representations of a *-algebra $\mathfrak{A}$ on Hilbert spaces $\mathfrak{H}_{\alpha}$. We denote the direct sum of these representations by $\pi=\bigoplus_{\alpha \in I_{0}} \pi_{\alpha}$ and define $\pi$ as follows. Let $\mathfrak{H}=\bigoplus_{\alpha \in I_{0}} \mathfrak{H}_{\alpha}$ be the direct sum of the Hilbert spaces $\mathfrak{H}_{\alpha}$ and let

$$
\begin{aligned}
\mathfrak{D}(\pi)= & \left\{F \sim\left\{F_{\alpha} ; \alpha \in I_{0}\right\} \in \mathfrak{H} ; F_{\alpha} \in \mathfrak{D}\left(\pi_{\alpha}\right) \text { for all } \alpha \in I_{0}\right. \text { and } \\
& \left.\sum_{\alpha \in I_{0}}\left\|\pi_{\alpha}(A) F_{\alpha}\right\|^{2}<\infty \text { for all } A \in \mathfrak{U}\right\} .
\end{aligned}
$$

We define $\pi(A) F=\pi(A)\left\{F_{\alpha} ; \alpha \in I_{0}\right\}=\left\{\pi_{\alpha}(A) F_{\alpha} ; \alpha \in I_{0}\right\}$ for all $F \in \mathfrak{D}(\pi)$ and $A \in \mathfrak{A}$. It is easily seen that $\pi$ is a closed *-representation of $\mathfrak{A}$ on $\mathfrak{H}$. Furthermore, $\pi$ is self-adjoint if and only if $\pi_{\alpha}$ is self-adjoint for all $\alpha \in I_{0}$. If $\mathfrak{A}$ is a commutative *-algebra then $\pi=\bigoplus_{\alpha \in I_{0}} \pi_{\alpha}$ is standard if and only if $\pi_{\alpha}$ is standard for all $\alpha \in I_{0}$.

Theorem 7.5. Suppose $\mathfrak{A}$ is a commutative *-algebra which is dominated by a countably generated *-subalgebra $\mathfrak{Q}_{1}$. Suppose $\pi$ is a self-adjoint representation of $\mathfrak{A}$. Then, $\pi$ is standard if and only if $\pi$ is the direct sum of strongly cyclic strongly positive self-adjoint representations of $\mathfrak{A}$.

Proof. From Theorem 7.3 it follows that if $\pi$ is the direct sum of strongly positive strongly cyclic self-adjoint representations of $\mathfrak{A}$ then $\pi$ is the direct sum of standard representations of $\mathfrak{U}$. Hence, $\pi$ is standard.

Conversely, suppose $\pi$ is standard. Since every *-representation of a $C^{*}$-algebra can be expressed as a direct sum of cyclic representations, there are vectors $\left\{f_{\alpha} \in \mathfrak{H}:\left\|f_{\alpha}\right\|=1, \alpha \in I_{0}\right\}$ such that with $\mathfrak{M}_{\alpha}=\left\{\overline{\pi(\mathfrak{Q})^{\prime \prime} f_{\alpha}}\right\}$, 
$\mathfrak{M}_{\alpha}$ is orthogonal to $\mathfrak{M}_{\beta}$ for $\alpha \neq \beta$ and $\mathfrak{H}=\bigoplus_{\alpha \in I_{0}} \mathfrak{M}_{\alpha}$ (i.e. $\pi(\mathfrak{U})^{\prime \prime}$ can be expressed as a direct sum of cyclic representations of $\left.\pi(\mathfrak{U})^{\prime \prime}\right)$.

Let $E_{\alpha}$ be the hermitian projection of $\mathfrak{H}$ onto $\mathfrak{M}_{\alpha}$. Clearly, $E_{\alpha} \in \pi(\mathfrak{U})^{\prime}$. By Theorem $4.4 \mathfrak{N}_{\alpha}=E_{\alpha} \mathfrak{D}(\pi)$ is a self-adjoint reducing subspace for $\pi$. Let $\pi_{\alpha}=\pi \mid \mathfrak{N}_{\alpha}$. It is easily verified that $\pi=\bigoplus \pi_{\alpha}$. Since $\pi$ is standard we have that $\pi_{\alpha}$ is standard in all $\alpha \in I_{0}$. Since $\frac{\alpha \in I_{0}}{\left\{\pi(\mathfrak{Y})^{\prime \prime} f_{\alpha}\right\}}=\mathfrak{M}_{\alpha}, f_{\alpha}$ is cyclic for $\pi_{\alpha}(\mathfrak{U})^{\prime \prime}$. Hence, by Lemma $7.4 \pi_{\alpha}$ is a strongly cyclic standard representation of $\mathfrak{A}$. Then, it follows from Theorem 4.7 that $\pi_{\alpha}$ is a strongly cyclic strongly positive self-adjoint representation of $\mathfrak{A}$. Hence, $\pi$ is a direct sum of strongly cyclic, strongly positive, self-adjoint representations of $\mathfrak{U}$. This completes the proof of the theorem.

\section{Standard Representations of the Canonical Commutation Relations}

Let $\mathfrak{U}_{0}$ be the ${ }^{*}$-algebra constructed in Example 2 of Section $\mathrm{V}$, i.e. $\mathfrak{U}_{0}$ consists of all polynomials in $\left\{p_{i}, q_{i} ; i, j=1, \ldots, s\right\}$ where the $q$ 's and $p$ 's satisfy the relations,

$$
\begin{aligned}
q_{i}^{*} & =q_{i} \quad p_{i}^{*}=p_{i}, \\
{\left[q_{i}, p_{j}\right] } & =q_{i} p_{j}-p_{j} q_{i}=i \delta_{i j} I, \\
{\left[q_{i}, q_{j}\right] } & =\left[p_{i}, p_{j}\right]=0
\end{aligned}
$$

for all $i, j=1, \ldots, s$. A representation $\pi$ of $\mathfrak{U}_{0}$ is called standard if $\pi$ is a direct sum of Schrodinger representations, i.e., $\pi=\bigoplus_{\alpha \in I_{0}} \pi_{\alpha}$ where $\pi_{\alpha}$ is unitarily equivalent to the Schrodinger representation (discussed in Example 2 of Section V). It follows that standard representations of $\mathfrak{A}_{0}$ are self-adjoint.

Definition 8.1. Suppose $\omega$ is a state of $\mathfrak{U}_{0}$ and $\pi_{0}$ is the Schrodinger representation of $\mathfrak{A}_{0}$ discussed in Example 2 of Section $\mathrm{V}$. We say $\omega$ is strongly positive if $\pi_{0}(A) \geqq 0$ implies $\omega(A) \geqq 0$ for all $A \in \mathfrak{A}_{0}$. If $\pi$ is a *-representation of $\mathfrak{U}_{0}$ we say $\pi$ is strongly positive if $\pi_{0}(A) \geqq 0$ implies $\pi(A) \geqq 0$ for all $A \in \mathfrak{U}_{0}$.

Using a result of Sherman [11] we show that every strongly positive strongly cyclic self-adjoint representation of $\mathfrak{U}_{0}$ is standard. We will need the following lemma.

Lemma 8.2. Suppose $\pi$ is a standard representation of $\mathfrak{U}_{0}$ on a Hilbert space $\mathfrak{H}$ and $\pi_{1}$ is a self-adjoint subrepresentation of $\pi$. Then $\pi_{1}$ is standard.

Proof. Suppose $\pi$ is a standard representation of $\mathfrak{U}_{0}$ on a Hilbert space $\mathfrak{H}$ and $\pi_{1}$ is a self-adjoint subrepresentation of $\pi$. By Theorem 4.7 
we have $\mathfrak{D}\left(\pi_{1}\right)=E \mathfrak{D}(\pi)$ and $\pi_{1}(A) f=\pi(A) f$ for all $A \in \mathfrak{A}_{0}$ and all $f \in \mathfrak{D}\left(\pi_{1}\right)$ where $E \in \pi\left(\mathfrak{U}_{0}\right)^{\prime}$ is an hermitian projection.

Since $\pi$ is standard we have $\pi=\bigoplus_{\alpha \in I_{0}} \pi_{\alpha}$ and $\mathfrak{H}=\bigoplus_{\alpha \in I_{0}} \mathfrak{H}_{\alpha}$ where $\pi_{\alpha}=\pi_{0}$ and $\mathfrak{H}_{\alpha}=\mathfrak{G}_{0}$ for all $\alpha \in I_{0}$, where $\pi_{0}$ is the Schrodinger representation of $\mathfrak{A}_{0}$ on $\mathfrak{H}_{0}=L^{2}\left(R^{s}\right)$.

The vector $f_{0}(x)=\pi^{-s / 4} e^{-\frac{1}{2}|x|^{2}}$ is strongly cyclic in the Schrodinger representation. This follows from the fact that the functions $p(x) f_{0}(x)$, where $p(x)$ is a polynomial are dense in Schwartz's space $\mathscr{S}\left(R^{s}\right)$ in the Schwartz space topology. Hence, $\left\{\pi_{0}\left(\mathfrak{A}_{0}\right) f_{0}\right\}$ is dense in $\mathfrak{D}\left(\pi_{0}\right)$ in the induced topology. Hence, $f_{0}$ is strongly cyclic for $\pi_{0}$. Furthermore, $\pi_{0}\left(i p_{i}+q_{i}\right) f_{0}=0$ for $i=1, \ldots, s$ and if $g \in \mathfrak{D}\left(\pi_{0}\right)$ and if $\pi_{0}\left(i p_{i}+q_{i}\right) g=0$ for $i=1, \ldots, s$ then $g=\alpha f_{0}$ for some complex number $\alpha$.

Since $\pi_{\alpha}$ and $\pi_{0}$ are unitarily equivalent there is a strongly cyclic vector $f_{\alpha} \in \mathfrak{D}\left(\pi_{\alpha}\right) \subset \mathfrak{H}_{\alpha}$ corresponding to the cyclic vector $f_{0} \in \mathfrak{D}\left(\pi_{0}\right) \subset \mathfrak{H}_{0}$, i.e., $\left\|f_{\alpha}\right\|=1, \pi_{\alpha}\left(i p_{i}+q_{i}\right) f_{\alpha}=0$ for $i=1, \ldots, s$. Note that $\left(f_{0}, \pi_{0}(A) f_{0}\right)$ $=\left(f_{\alpha}, \pi_{\alpha}(A) f_{\alpha}\right)$ for all $A \in \mathfrak{A}_{0}$ and $\alpha \in I_{0}$. Let $\mathfrak{M}_{0}$ be the closed span of $\left\{f_{\alpha} ; \alpha \in I_{0}\right\}$. We claim $\mathfrak{M}_{0} \subset \mathfrak{D}(\pi)$ and $f \in \mathfrak{M}_{0}$ if and only if $f \in \mathfrak{D}(\pi)$ and $\pi\left(i p_{i}+q_{i}\right) f=0$ for $i=1, \ldots, s$.

Suppose $f \in \mathfrak{M}_{0}$. Let $E_{\alpha}$ be the hermitian projection of $\mathfrak{Y}$ onto $\mathfrak{S}_{\alpha}$. Since $f \in \mathfrak{M}_{0}$ there is a sequence $\left\{f_{n} ; n=1,2, \ldots\right\}$ such that $f_{n}$ is a finite linear combination of $f_{\alpha}$ for each $n$ and $f_{n} \rightarrow f$ as $n \rightarrow \infty$. Note that if $f_{n}=\sum_{\alpha \in I_{0}} c_{n}(\alpha) f_{\alpha}$ we have

$$
\begin{aligned}
\left\|E_{\alpha} \pi(A)\left(f_{n}-f_{m}\right)\right\|^{2} & =\left\|\pi(A) E_{\alpha}\left(f_{n}-f_{m}\right)\right\|^{2} \\
& =\left|c_{n}(\alpha)-c_{m}(\alpha)\right|^{2}\left\|\pi_{\alpha}(A) f_{\alpha}\right\|^{2} \\
& =\left\|E_{\alpha}\left(f_{n}-f_{m}\right)\right\|^{2}\left\|\pi_{0}(A) f_{0}\right\|^{2} .
\end{aligned}
$$

Hence, we have

$$
\begin{aligned}
\left\|\pi(A) f_{n}-\pi(A) f_{m}\right\|^{2} & =\sum_{\alpha \in I_{0}}\left\|E_{\alpha} \pi(A)\left(f_{n}-f_{m}\right)\right\|^{2} \\
& =\left\|\pi_{0}(A) f_{0}\right\|^{2} \sum_{\alpha \in I_{0}}\left\|E_{\alpha}\left(f_{n}-f_{m}\right)\right\|^{2} \\
& =\left\|\pi_{0}(A) f_{0}\right\|^{2}\left\|f_{n}-f_{m}\right\|^{2} \rightarrow 0
\end{aligned}
$$

as $n, m \rightarrow \infty$. Since $A \in \mathfrak{U}_{0}$ is arbitrary we have $\left\{f_{n} ; n=1,2, \ldots\right\}$ is a Cauchy sequence in the induced topology on $\mathfrak{D}(\pi)$. Since $f_{n} \rightarrow f$ as $n \rightarrow \infty$ and $\pi$ is closed we have $f \in \mathfrak{D}(\pi)$. Since $\pi\left(i p_{i}+q_{i}\right) f_{n}=0$ for $i=1, \ldots, s$ and $n=1,2, \ldots$ we have $\pi\left(i p_{i}+q_{i}\right) f=0$ for $i=1, \ldots, s$. 
Conversely, suppose $f \in \mathfrak{D}(\pi)$ and $\pi\left(i p_{i}+q_{i}\right) f=0$ for $i=1, \ldots, s$. Then, since $E_{\alpha} \in \pi\left(\mathfrak{A}_{0}\right)^{\prime}$ we have

$$
E_{\alpha} \pi\left(i p_{i}+q_{i}\right) f=\pi\left(i p_{i}+q_{i}\right) E_{\alpha} f=0
$$

for $i=1, \ldots, s$. Hence, we have $E_{\alpha} f=c(\alpha) f_{\alpha}$ where $c(\alpha)$ is a complex number. Hence, $f \in \mathfrak{M}_{0}$.

We recall that $\mathfrak{D}\left(\pi_{1}\right)=E \mathfrak{D}(\pi)$ where $E \in \pi\left(\mathfrak{A}_{0}\right)^{\prime}$ is an hermitian projection. Let $\mathfrak{N}=E \mathfrak{M}_{0}$. We show $\mathfrak{N} \subset \mathfrak{M}_{0}$. Suppose $f \in \mathfrak{M}_{0}$. Then $\pi\left(i p_{i}+q_{i}\right) E f=E \pi\left(i p_{i}+q_{i}\right) f=0$ for $i=1, \ldots, s$. Hence, $E f \in \mathfrak{M}_{0}$ and $\mathfrak{N} \subset \mathfrak{M}_{0}$.

Let $\left\{g_{\beta} ; \beta \in I_{1}\right\}$ be an orthonomal basis for $\mathfrak{N}$ and let $\mathfrak{N}_{\beta}=\left\{\pi\left(\mathfrak{A}_{0}\right) g_{\beta}\right\}$. We claim $\left(g_{\beta}, \pi(A) g_{\beta^{\prime}}\right)=\delta_{\beta \beta^{\prime}}\left(f_{0}, \pi_{0}(A) f_{0}\right)$ for all $A \in \mathfrak{A}_{0}$ and $\beta, \beta^{\prime} \in I_{1}$. Since $g_{\beta}, g_{\beta^{\prime}} \in \mathfrak{M}_{0}$ we have $g_{\beta}=\sum_{\alpha \in I_{0}} c(\alpha) f_{\alpha}$ and $g_{\beta^{\prime}}=\sum_{\alpha \in I_{0}} c^{\prime}(\alpha) f_{\alpha^{\prime}}$. Then, we have

$$
\begin{aligned}
\left(g_{\beta}, \pi(A) g_{\beta^{\prime}}\right) & =\sum_{\alpha \in I_{0}}\left(g_{\beta_{1}} E_{\alpha} \pi(A) g_{\beta^{\prime}}\right) \\
& =\sum_{\alpha \in I_{0}}\left(E_{\alpha} g_{\beta}, \pi(A) E_{\alpha} g_{\beta^{\prime}}\right) \\
& =\sum_{\alpha \in I_{0}} \overline{c(\alpha)} c^{\prime}(\alpha)\left(f_{\alpha}, \pi_{\alpha}(A) f_{\alpha}\right) \\
& =\left(g_{\beta}, g_{\beta^{\prime}}\right)\left(f_{0}, \pi_{0}(A) f_{0}\right)=\delta_{\beta \beta^{\prime}}\left(f_{0}, \pi_{0}(A) f_{0}\right) .
\end{aligned}
$$

Hence, we have $\mathfrak{N}_{\beta}{ }^{\perp} \mathfrak{N}_{\beta^{\prime}}$ for $\beta \neq \beta^{\prime}$. Let $\pi_{\beta}$ be the closure of the restriction of $\pi$ to $\mathfrak{N}_{\beta}$, i.e. $\pi_{\beta}=\left(\pi \mid \mathfrak{N}_{\beta}\right)$. $\pi_{\beta}$ is unitarily equivalent to $\pi_{0}$ since $f_{0}$ and $g_{\beta}$ are strongly cyclic for $\pi_{0}$ and $\pi_{\beta}$ and $\left(f_{0}, \pi_{0}(A) f_{0}\right)=\left(f_{\beta}, \pi_{\beta}(A) f_{\beta}\right)$ for all $A \in \mathfrak{U}_{0}$.

Let $\pi_{2}=\bigoplus_{\beta \in I_{1}} \pi_{\beta}$. Since $\pi_{2}$ is the direct sum of representation which are unitarily equivalent to $\pi_{0}, \pi_{2}$ is standard. We complete the proof of this lemma by showing $\pi_{2}=\pi_{1}$.

Since $\pi_{2}$ is a self-adjoint subrepresentation of $\pi$ it follows from Theorem 4.7 that $\mathfrak{D}\left(\pi_{2}\right)=F \mathfrak{D}(\pi)$ with $F \in \pi\left(\mathfrak{A}_{0}\right)^{\prime}$ an hermitian projection. We show $\pi_{1}=\pi_{2}$ by showing $E=F$. Since $\mathfrak{N}_{\beta} \subset \mathfrak{D}\left(\pi_{1}\right)$ for all $\beta \in I_{1}$, we have $\pi_{1} \supset \pi_{2}$ and, therefore, $E \geqq F$. We show $F \geqq E$. Suppose $f \in\{E \mathfrak{S}\}$ and $\varepsilon>0$. Since $\mathfrak{D}\left(\pi_{1}\right)$ is dense in $\{E \mathfrak{S}\}$ there is a $g \in \mathfrak{D}\left(\pi_{1}\right)$ with $\|f-g\|$ $<\varepsilon / 2$. Since $f_{\alpha}$ is cyclic for $\pi_{\alpha}$ and $\pi=\bigoplus_{\alpha \in I_{0}} \pi_{\alpha}$ it follows that the linear span of $\left\{\pi\left(\mathfrak{A}_{0}\right) f_{\alpha} ; \alpha \in I_{0}\right\}$ is dense in $\mathfrak{H}$. Hence, there is a vector $h$ of the form $h=\sum_{n=1}^{m} \pi\left(A_{n}\right) f_{\alpha_{n}}$ with $A_{n} \in \mathfrak{A}_{0}$ and $\|h-g\|<\varepsilon / 2$. Let

$$
k=\sum_{n=1}^{m} \pi\left(A_{n}\right) E f_{\alpha_{n}}=E h .
$$


Since $g \in \mathfrak{D}\left(\pi_{1}\right) \subset\{E \mathfrak{S}\}$ we have $\|g-k\|=\|E g-E h\| \leqq\|g-h\|<\varepsilon / 2$. Hence, we have

$$
\|f-k\| \leqq\|f-g\|+\|g-k\|<\varepsilon / 2+\varepsilon / 2=\varepsilon .
$$

We claim $k \in\{F \mathfrak{H}\}$. We show $E f_{\alpha} \in\{F \mathfrak{H}\}$ for all $\alpha \in I_{0}$. Since $f_{\alpha} \in \mathfrak{M}_{0}$, $E f_{\alpha} \in \mathfrak{N}$. We have $g_{\beta} \in \mathfrak{D}\left(\pi_{2}\right) \subset\{F \mathfrak{H}\}$ for all $\beta \in I_{1}$. Since, the $\left\{g_{\beta}\right\}$ span $\mathfrak{N}$ we have $E f_{\alpha} \in \mathfrak{N} \subset\{F \mathfrak{S}\}$. Hence, $E f_{\alpha} \in\{F \mathfrak{H}\}$ for all $\alpha \in I_{0}$. Hence, $k \in\{F \mathfrak{H}\}$, since

$$
\begin{aligned}
F k & =\sum_{n=1}^{m} F \pi\left(A_{n}\right) E f_{\alpha_{n}} \\
& =\sum_{n=1}^{m} \pi\left(A_{n}\right) F E f_{\alpha_{n}} \\
& =\sum_{n=1}^{m} \pi\left(A_{n}\right) E f_{\alpha_{n}}=k .
\end{aligned}
$$

Hence, each $f \in\{E \mathfrak{H}\}$ can be approximated arbitrarily well by a $k \in\{F \mathfrak{H}\}$. Hence, $F \geqq E$. Therefore, $E=F$ and $\pi_{1}=\pi_{2}$. Hence, $\pi_{1}$ is standard and the proof is complete.

Sherman [11] has proved the following useful result. Suppose $\mathfrak{A}$ is a countably generated *-algebra and $\pi_{1}$ is a closed *-representation of $\mathfrak{U}$ on a Hilbert space $\mathfrak{S}$ such that there is an $A_{0} \in \mathfrak{A}$ with the property that $\pi_{1}\left(A_{0}\right)$ is the restriction to $\mathfrak{D}\left(\pi_{1}\right)$ of the inverse of a compact operator. Then, if $\omega$ is a state of $\mathfrak{U}$ with the property that $\omega(A) \geqq 0$ for all $A \in \mathfrak{A}$ with $\pi_{1}(A) \geqq 0$, then $\omega$ is of the form

$\omega(A)=\sum^{\infty}\left(f_{i}, \pi_{1}(A) f_{i}\right)$ for all $A \in \mathfrak{A}$ with $f_{i} \in \mathfrak{D}\left(\pi_{1}\right)$ for $i=1,2, \ldots$.

From Sherman's theorem we derive the following result.

Theorem 8.3. Suppose $\pi$ is a strongly cyclic self-adjoint representation of $\mathfrak{A}_{0}$ on a Hilbert space $\mathfrak{H}$ with a strongly cyclic vector $f_{0} \in \mathfrak{D}(\pi)$ with $\left\|f_{0}\right\|=1$. Let $\omega(A)=\left(f_{0}, \pi(A) f_{0}\right)$ for $A \in \mathfrak{A}_{0}$ be the state determined by $f_{0}$. Then, $\pi$ is standard if and only if $\omega$ is strongly positive.

Proof. Suppose $\pi$ is standard. Then $\pi$ is a direct sum of Schrodinger representations and, therefore, $\pi$ is strongly positive. Hence, $\omega$ is strongly positive.

Conversely, suppose $\omega$ is strongly positive. In the Schrodinger representation $\pi_{0}, \overline{\pi_{0}(H)}$ with $H=\sum_{i=1}^{s} p_{i}^{2}+q_{i}^{2}$ is well known to have point spectrum with finite multiplicities (in fact, $\left.\sigma \overline{\left(\pi_{0}(H)\right.}\right)=\{s+2 n ; n=0,1,2, \ldots\}$ ). Hence, $\overline{\pi_{0}(H)}{ }^{-1}$ is compact. Since $\omega$ is strongly positive (positive relative 
to the Schrodinger representation) it follows from a theorem of Sherman [11] that $\omega$ is of the form

$$
\omega(A)=\sum_{i=1}^{n}\left(f_{i}, \pi_{0}(A) f_{i}\right)
$$

for all $A \in \mathfrak{A}_{0}$, with $f_{i} \in \mathfrak{D}\left(\pi_{0}\right)$ for $i=1,2, \ldots$.

Let $\pi_{1}=\bigoplus_{n=1}^{\infty} \pi_{0}$ be the direct sum of $\pi_{0}$ countably infinitely many times. Let $\mathfrak{H}_{1}=\bigoplus_{n=1}^{\infty} \mathfrak{H}_{0}$ be the representation space of $\pi_{1}$. Let $f \sim\left\{f_{1}, f_{2}, \ldots\right\}$ be the vector in $\mathfrak{H}_{1}$ with components $f_{i} \in \mathfrak{D}\left(\pi_{0}\right)$. Since $f_{i} \in \mathfrak{D}\left(\pi_{0}\right)$ for $i=1,2, \ldots$ and $\sum_{i=1}^{\infty}\left\|\pi_{0}(A) f_{i}\right\|^{2}=\omega\left(A^{*} A\right)<\infty$ for all $A \in \mathfrak{U}_{0}$, it follows that $f \in \mathfrak{D}\left(\pi_{1}\right)$. Note $\omega(A)=\left(f, \pi_{1}(A) f\right)$ for all $A \in \mathfrak{A}_{0}$.

Let $\mathfrak{N}=\left\{\pi_{1}\left(\mathfrak{Q}_{0}\right) f\right\}$ and let $\pi_{2}$ be the closure of the restriction of $\pi_{1}$ to $\mathfrak{N}$, i.e., $\pi_{2}=\left(\pi_{1} \mid \mathfrak{P}\right) . \pi_{2}$ is unitarily equivalent to $\pi$ since $f$ and $f_{0}$ are strongly cyclic for $\pi_{2}$ and $\pi$ and

$$
\left(f, \pi_{2}(A) f\right)=\omega(A)=\left(f_{0}, \pi(A) f_{0}\right)
$$

for all $A \in \mathfrak{A}_{0}$. Since $\pi$ is self-adjoint $\pi_{2}$ is self-adjoint. Hence, $\pi_{2}$ is a selfadjoint restriction of $\pi_{1}$. Since $\pi_{1}$ is standard it follows from Lemma 8.2 that $\pi_{2}$ is standard. Since $\pi$ and $\pi_{2}$ are unitarily equivalent we have $\pi$ is standard. This completes the proof of the theorem.

\section{References}

1. Bourbaki, N.: Éléments de mathématiques Livre V, Espaces vectoriels topologiques. Paris: Herman 1955.

2. Dixmier, J.: Les $C^{*}$-algébras et leurs représentations. Paris: Gauthier-Villars 1964.

3. Dunford, N., Schwartz, J. T.: Linear operators part II. New York: Interscience Pub. 1963.

4. Gelfand, M., Vilenkin, N. Ya.: Generalized functions, Vol. IV. New York: Academic Press 1964.

5. Jaffe, A. M.: Dynamics of a cut-off $\lambda \phi^{4}$ field theory. Thesis Princeton University (1965).

6. Naimark, M. A.: Normed Rings. Groningen, The Netherlands: P. Noordholf N. V. 1964.

7. Nelson, E.: Analytic vectors. Ann. Math. 70, 572-615 (1959).

8. Reed, M.: A garding domain for quantum fields. Commun. math. Phys. 14, 336-346 (1969).

9. Segal I. E.: Irreducible representations of operator algebras. Bull. Am. Math. Soc. 53, $73-88$ (1947).

10. - Decomposition of operator algebras, II. Memoirs of the American Mathematical Society, No. 9, New York (1951).

11. Sherman, T.: Positive linear functionals on *-algebras of unbounded operators. J. Math. Anal. Appl. 22, 285-318 (1968). 
12. Shohat, J. A., Tamarkin, J. D.: The problem of moments, surveys, No. 1. Am. Math. Soc. New York (1943).

13. Simon, B.: Distributions and their Hermite expansions. Preprint.

14. Stone, M. H.: Linear transformation in Hilbert space and their applications to analysis. Am. Math. Soc. Colloquium Pub., Vol. 15, New York (1932).

15. Streater, R. F., Wightman, A. S.: P.C.T., spin and statistics and all that. New York: Benjamin 1964.

16. Riesz, F., Nagy, B. Sz.: Functional analysis. New York: Fredrich Ungar Pub. 1955.

17. Neumann, J. von: Die Eindeutigkeit der Schrödingerschen Operatoren. Math. Ann. 104, 570-578 (1931).

\author{
Robert T. Powers \\ University of Pennsylvania \\ Philadelphia, Pa. 19104, USA \\ Present address: \\ School of Natural Sciences \\ The Institute of Advanced Study \\ Princeton, New Jersey 08540, USA
}

